Preprints of the

Max Planck Institute for

Research on Collective Goods

Bonn 2006/15

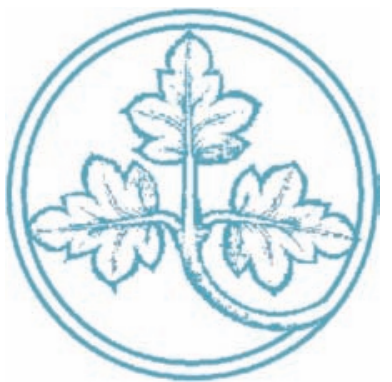

Monetary Equilibria in a Baumol-Tobin Economy

Ingolf Schwarz

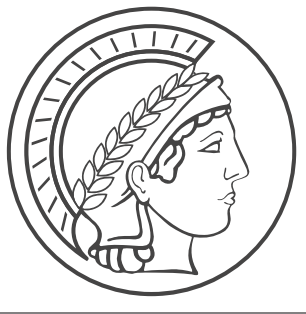




\section{Monetary Equilibria in a Baumol-Tobin Economy}

Ingolf Schwarz

June 2006 


\title{
Monetary Equilibria in a Baumol-Tobin Economy
}

\author{
Ingolf Schwarz* \\ Max Planck Institute for Research on Collective Goods, Bonn \\ and \\ CDSEM, University of Mannheim
}

30th March 2006

\begin{abstract}
This paper provides a non-steady state general equilibrium foundation for the transactions demand for money going back to Baumol (1952) and Tobin (1956). In our economy, money competes against real capital as a store of value. We prove existence of a monetary general equilibrium in which both real capital and fiat money are voluntarily held over time. The demand for money is generated by fixed transactions costs. More precisely, we assume that households have two physically separated accounts. On the first account they finance consumption and might want to hold money over time. On the second account households receive their wages, hold claims on capital and earn interest income from renting capital to firms. Every transfer of wealth between the two accounts requires fixed resources. In equilibrium, households space apart the transaction dates in time. Between these transaction dates, money is held as a store of value on the first account for transactions purposes. The number of periods over which money is held is endogenous and the nonconvexity of the problem is explicitly taken into account.

Key Words: Baumol-Tobin, Monetary Theory, General Equilibrium Theory

JEL Classification Numbers: D50, E40, E41
\end{abstract}

\footnotetext{
${ }^{*}$ Max Planck Institute for Research on Collective Goods, Kurt-Schumacher-Str. 10, 53113 Bonn, Germany. Email: schwarz@coll.mpg.de. This paper would not exist in this form without the ongoing support of Martin Hellwig and the fruitful discussions with him. I would also like to thank Jinhui Bai, Felix Bierbrauer, Hendrik Hakenes and the participants in the seminar at the Max Planck Institute for Research on Collective Goods in Bonn and in the workshop for mathematical economics at Yale for helpful comments. Financial support from the Deutsche Forschungsgemeinschaft is gratefully acknowledged.
} 


\section{Introduction}

We model a dynamic competitive economy in which fiat money competes against real capital as a store of value. The main result of the paper is the existence of general equilibria in which the value of money is bounded away from zero. In every such equilibrium both capital and money are used as stores of value. The driving force behind this result is not one of the frictions usually used in monetary economies, e.g. cash-in-advance or money in the utility function. We rather derive our general equilibrium result in the spirit of Baumol (1952) and Tobin (1956) by giving money a transactions costs advantage over real capital. By way of introduction, we subsequently motivate the study by explaining the relevant doctrinal history before we then sum up the structure of the model.

In his classical contribution, Frank Hahn (1965) considered a standard Walrasian finite horizon economy in which outside money can only be valued to the extend that it is exchanged for something else. He proved the negative result that, under an assumption which involves that the utility of money is zero if money holdings itself is zero, the existence of a Walrasian equilibrium does not imply the existence of a monetary Walrasian equilibrium. There is no mechanism which prevents that the value of money may be driven out. This result has become known as the Hahn problem. ${ }^{1}$

One possibility to get rid of the Hahn problem is to model money as a store of value. For instance, in a standard overlapping-generations framework, Grandmont and Laroque (1973) argued that money serves as the store of value to finance consumption after retirement. Bewley $(1980,1983)$ and Hellwig (1982) model economies where money is held for precautionary reasons as a hedge against stochastic shocks in the value of households endowments. Driven by the convexity of preferences, the same structural result is also true with purely deterministic fluctuations in income. ${ }^{2}$

In these papers, however, money is assumed to be the only store of value in the economy. The main result of the analysis, namely that money has value because it is held over time, becomes wrong if worthless fiat money has to compete in a frictionless economy against another asset which dominates money in the return characteristics. Even stronger, if there exists an asset next to money having a higher own rate of return than money but the

\footnotetext{
${ }^{1}$ If money does not enter non-satiated utilities at all, then every equilibrium must involve a zero value of money. This stronger formulation of the Hahn problem is used in most citations in the literature.

${ }^{2}$ Of course, this does not give rise to precautionary money demand. There is rather a demand for a store of value to smooth consumption over time.
} 
same marketability properties, then the value of money must be zero in every rational expectations environment (Hahn (1982), Hellwig (1993)). This problem is called the modified Hahn problem (Hellwig (1993)).

To derive a positive value of money in the presence of dominant assets, the latter result implies that money must have advantages in the marketability properties as compared to the other assets. The first and easiest possibility to achieve this is to model a cash-in-advance constraint. A second possibility, which we will employ in this paper, is to introduce fixed transaction costs. The seminal contribution following this idea is Gale and Hellwig $(1984,1988)$. Their model economy is populated by a continuum of identical and infinitely lived households and each household owns and manages its own firm. Money competes as a store of value against capital which can be invested in the firms. Assuming that the payments of dividends between the agent's firm account and the agent's consumption account require fixed real costs implies that money survives as a store of value. Indeed, it is held over time to finance consumption between the transaction dates.

To use transaction costs as the driving force for money demand in the presence of dominant assets goes back to Baumol's (1952) and Tobin's (1956) analysis of the transactions demand for money. They basically argued that wealth in the form of the interest bearing asset must first be transferred at fixed transactions costs to the money purse, say, before it can be used to buy consumption goods. Since money can directly be held in the money purse, it has an advantage in its marketability characteristics. However, the analysis of Baumol and Tobin does not provide a positive answer to the modified Hahn problem because it is not an equilibrium analysis. They focus on an isolated optimization problem of an agent minimizing his cost of cash management over time. ${ }^{3}$ The coordination of these microeconomic plans in the markets, i.e. whether the analysis is consistent with market clearing, is not analyzed at all. In fact, Baumol and Tobin develop a microeconomic decision problem taking the value of money for granted and assuming a constant an exogenous interest rate. Furthermore, the analysis of individual optimization has deep conceptual problems. The most important critical points are that the analysis is conducted in terms of the time averages of the decision variables instead of the decision variables themselves and that the nonconvexity of the problem is just assumed away. ${ }^{4}$ It is clear that a model which proves the validity of Baumol's and Tobin's argument in a general

\footnotetext{
${ }^{3}$ These costs are opportunity costs from holding money since there is an asset with a higher rate of return plus transaction costs which arise from a transfer of asset market wealth to the money purse.

${ }^{4}$ A detailed discussion of these issues can be found in Hellwig (1993).
} 
equilibrium corresponds to a solution of the modified Hahn problem.

Related Literature. There is quite a large literature on Baumol-Tobin in general equilibrium models focusing on steady-states. In such models, the problem of market clearing mentioned above, i.e. the coordination of individual plans in the markets, does not arise by construction. Papers contributing to this literature are Romer (1986), Jovanovic (1982), Grossman (1987), Chatterjee and Corbae (1992), Grossman and Weiss (1983), Rotemberg (1984) or Zeira (2005). They restrict attention to steady-state equilibria in which the problem of missing trading partners is constructed away, but cannot be considered as solved. A stylized example could be as follows. Suppose we have a two period overlapping-generations framework in which agents start their life with an endowment of money, invest, say, one half of it in the bond and spend the other half on consumption when they are young. Once retired, they need to sell their bonds for cash to finance consumption when they are old. At the same instant of time when the young generation turns to get old, a new generation with equal characteristics is born which just desires as many bonds as the older generation supplies. This construction allows for a constant interest rate and a steady-state equilibrium. There is no trade in bonds or money at other instants of time. However, a steady-state analysis where trade is permitted only occasionally to make a model work should not be considered as an exhausting analysis of this topic. Another disadvantage of this literature is that the nonconvexity is mostly neglected or absent by construction. ${ }^{5}$

Non-steady-state models are Gale and Hellwig (1984, 1988), Shubik and Yao (1993) or Dréze and Polemarchakis (2000). Dréze and Polemarchakis (2000) use a finite horizon framework with inside money only. They do not address the equilibrium problem mentioned before because they refer to time averages and a constant and exogenous interest rate within a single BaumolTobin period. Shubik and Yao (1993) model exogenous interest rates and rely on an analysis in terms of time averages. The latter implies that the whole structure "within the period" is left open.

Outline of the Model. The model presented here basically builds on the insights of Gale and Hellwig $(1984,1988)$ and extends their model. Time is discrete, the horizon is unbounded and the economy is populated by house-

\footnotetext{
${ }^{5}$ Romer(1986), Grossman (1987), Grossman and Weiss (1983) or Rotemberg (1984) are examples for such an assumption. Grossman and Weiss (1983) and Rotemberg (1984) give a detailed steady-state analysis but restrict attention to exogenous transaction patterns of the households. That is, every household is assumed to sacrifice the fixed costs every second period, say. This allows them to construct a steady-state equilibrium and also to get rid of the nonconvexity.
} 
holds and firms. In every period, there is a labor market, a market for capital, a money market and a market for a physical output good. The latter can be consumed or invested. Households accumulate the claims on capital to earn a return from renting it to firms and they supply labor to earn wage income. A transactions demand for money is generated by the assumption that households have two physically separated accounts: a cash account and a checking account. On the latter account they accumulate claims on capital and earn their labor and rental income. On the cash account, households may choose to inventory money and to finance consumption. If households want to transfer wealth between their two accounts, they have to sacrifice some fixed costs because the two accounts are physically separated. These fixed costs eventually prevent the households from transferring wealth every period but rather provides for the incentives to hold money as a store of value on the cash account between two transfer periods.

The transaction patterns of households are endogenous. Equivalently, the number of periods over which households choose to hold money is determined by optimization. It can even be zero for a positive measure of households. The reason is that after financing a transfer today, no money holdings between yesterday and today is required to finance today's consumption. To put it differently, we not impose an assumption on the timing of markets. Because goods must be bought with money, but the time period over which money is held is not exogenously fixed, one can say that this model has a "flexible cash-in-advance constraint". ${ }^{6}$

In Section 2.2 we describe the economy, the households and firms. In Section 2.3 we define the equilibrium, in Section 2.4 we state our assumptions and the result of the paper. In Section 2.5 we summarize some properties of the equilibrium, in Section 2.6 we conclude the paper and in the appendix to this chapter, Section 2.7, we prove the result.

\section{The Model}

\subsection{The Basic Structure}

Time is discrete, a period is denoted with $t$ and the set of periods is $T:=\mathbb{N}$. The economy is populated by households and firms. The generic household is denoted with $h$ and the set of households, $H$, is without loss of generality

\footnotetext{
${ }^{6}$ In a typical cash-in-advance economy the number of periods over which an agent holds money is by construction exogenously fixed to one: Households receive their income in the evening of a day and need to hold it over night to use it for whatever they want at the next day.
} 
assumed to be the unit interval, $H=[0,1]$. There is a single physical output good which is produced by firms using labor and capital as inputs. Households supply labor every period to earn wage income. At each point in time, current output of the economy must be divided into gross investment in the capital stock and consumption. This consumption-saving decision is made by households. In addition, there is fiat money which can be held as a store of value. The generic firm is denoted with $j$ and the set of firms, $J$, is without loss of generality assumed to be the unit interval, $J=[0,1]$. Firms have identical production functions $F$ which transform labor and capital into the single output good of this economy. In every period, competitive labor, capital, output and money markets meet. The Lebesgue measure is assumed to be the population measure for both households and firms.

\subsection{Households}

The friction which generates a transactions demand of households for money is as follows. Every household $h \in H$ has two separated accounts, a cash account, or money purse, and a checking account. The latter can be thought of as being held in an unmodelled bank. Let $p_{t}$ denote the price of the output good in period $t$ in terms of money and $c_{t}^{h}$ denote consumption of $h \in H$ in period $t \in T$. $h$ must finance his consumption expenditure in period $t, p_{t} c_{t}^{h}$, on the cash account. To do so, he can either use cash inventoried voluntarily from the previous period, $m_{t-1}^{h}$, or alternatively use a transfer of nominal wealth from the checking account to the cash account, $\Delta_{t}^{h}$. Importantly, the household is not forced to hold any money over time as in a standard cash-in-advance model. Instead he can use the nominal income he receives in the same period on the checking account for consumption purposes.

In case of a nominal transfer between his two accounts, household $h$ has to sacrifice some fixed transactions costs. These transactions costs consist of transactions costs in the output good, $\gamma_{c}$, and transactions costs in time or labor, $\gamma_{l}$. As for the labor costs, the transactions costs mapping is denoted with $\kappa_{l}$ and defined by

$$
\kappa_{l}(\Delta):= \begin{cases}0 & \text { if } \Delta=0 \\ \gamma_{l} & \text { otherwise }\end{cases}
$$

For the output costs, we define the two mappings $\kappa_{c}^{1}$ and $\kappa_{c}^{2}$ by

$$
\kappa_{c}^{1}(\Delta)\left[\operatorname{resp} . \kappa_{c}^{2}(\Delta)\right]:= \begin{cases}\gamma_{c} & \text { if } \Delta<0[\operatorname{resp} . \Delta>0] \\ 0 & \text { otherwise. }\end{cases}
$$


If $\Delta_{t}^{h} \neq 0$ then the household has to "purchase $\gamma_{c}$ on the market" at costs $p_{t} \gamma_{c}$. We understand the direction of the transfer such that if $\Delta_{t}^{h}>0$ then household $h$ transfers wealth in period $t$ from the checking account to the cash account and vice versa for $\Delta_{t}^{h}<0$. The two mappings $\kappa_{c}^{1}$ and $\kappa_{c}^{2}$ are used to model the circumstance that the transactions costs are paid on the account from which the transfer is financed. So if $\Delta_{t}^{h}>0$, the household has to finance the expenses for the fixed costs, $p_{t} \kappa_{c}^{2}\left(\Delta_{t}^{h}\right)=p_{t} \gamma_{c}$, on the checking account and if $\Delta_{t}^{h}<0$, he has to finance $p_{t} \kappa_{c}^{1}\left(\Delta_{t}^{h}\right)=p_{t} \gamma_{c}$ on the cash account. ${ }^{7}$

Denote with $e$ the time invariant labor time endowment of every $h$. Since leisure is no argument of utilities, all the available time is supplied inelastically as labor as long as the wage is positive. Let $w_{t}$ denote the real wage, $r_{t}$ be the real rental rate for capital use between $t-1$ and $t$ and $k_{t}^{h} \geq 0$ be the claims on capital household $h$ owns between the periods $t-1$ and $t$. On the checking account, $h$ earns his wage income, which is $p_{t} w_{t}\left(e-\kappa_{l}\left(\Delta_{t}^{h}\right)\right)$ in nominal terms, he invests in the capital stock he wants to own at the end of period $t, i_{t}^{h} \gtreqless 0$, he receives the nominal return from renting the capital he owned between $t-1$ and $t$ to firms, $p_{t} k_{t}^{h} r_{t}$, and he finally receives nominal dividend payments from firms, $p_{t} D_{t}^{h}$. The law of motion for capital owned by household $h$ is linear and takes the usual form $k_{t+1}^{h}=i_{t}^{h}+(1-\delta) k_{t}^{h}$, where $\delta$ is the constant rate of depreciation, $0<\delta<1$. In period one, every household $h \in H$ is endowed with outside money $\bar{m}^{h}>0$ on the cash account and with capital $\bar{k}^{h}>0$ on the checking account. Let $m_{t}^{h} \geq 0$ be the money held by $h$ at the end of $t$ on the cash account and $n_{t}^{h} \geq 0$ be the money held by $h$ at the end of $t$ on the checking account.

Every household has one constraint for each account. The constraints in the first period on the cash account and on the checking account are, respectively,

$$
\begin{aligned}
m_{1}^{h}+p_{1} c_{1}^{h}+p_{1} \kappa_{c}^{1}\left(\Delta_{1}^{h}\right) & \leq \bar{m}^{h}+\Delta_{1}^{h} \\
n_{1}^{h}+p_{1} k_{2}^{h}+\Delta_{1}^{h}+p_{1} \kappa_{c}^{2}\left(\Delta_{1}^{h}\right) & \leq p_{1} w_{1}\left(e-\kappa_{l}\left(\Delta_{1}^{h}\right)\right)+p_{1} k_{1}^{h}\left(r_{1}+1-\delta\right)+p_{1} D_{1} \\
k_{1}^{h} & \leq \bar{k}^{h}
\end{aligned}
$$

\footnotetext{
${ }^{7}$ This structure is imposed to show that the demand sets of the households are upper hemi-continuous. If the household would be forced to finance the transactions costs in the output from a single account, no matter in which direction the transfer goes, then this property cannot be shown to be true for every price vector. However, this seems to be an artifact resulting from the assumption that there is no bond which the household can sell in order to borrow. So in a more general framework, we conjecture that one can dispense with this assumption.
} 
and in every period $t \geq 2$,

$$
\begin{aligned}
m_{t}^{h}+p_{t} c_{t}^{h}+p_{t} \kappa_{c}^{1}\left(\Delta_{t}^{h}\right) \leq & m_{t-1}^{h}+\Delta_{t}^{h} \\
n_{t}^{h}+p_{t} k_{t+1}^{h}+\Delta_{t}^{h}+p_{t} \kappa_{c}^{2}\left(\Delta_{t}^{h}\right) \leq & p_{t} w_{t}\left(e-\kappa_{l}\left(\Delta_{t}^{h}\right)\right) \\
& +p_{t} k_{t}^{h}\left(r_{t}+1-\delta\right)+n_{t-1}^{h}+p_{t} D_{t} .
\end{aligned}
$$

We will assume constant returns to scale which implies that the dividends are zero if the firms maximize. ${ }^{8}$ Notice that we have already plugged in the law of motion for capital to simplify the presentation.

The interpretation of the capital held in the checking account is as follows: in period $t \in T$, households choose to own some physical output not for consumption but for savings reasons. They rent this physical object to firms who will pay a rental rate for using it in $t+1$. This output to be saved is called capital and the quantity of capital owned by $h$ in $t$ is denoted with $k_{t+1}^{h}$. Households cannot hold this physical object themselves because they are not endowed with some kind of storage technology. Instead, they supply this capital in $t$ to firms to earn a revenue consisting of two terms in $t+1$ : first, the rents firms pay for using capital in production, $p_{t+1} k_{t+1}^{h} r_{t+1}$, and secondly the revenue from selling on the output market the ownership of what is left from their capital after firms have produced with it, $p_{t+1} k_{t+1}^{h}(1-\delta)$. Note that the physical object capital is held in the firm sector because they use it in the production process. They pay the rents for using this input in $t+1$, when the production process is finished, but capital supply and demand actually meet already in $t$.

Denote $p:=\left(p_{t}\right)_{t \in T}$ and use the same notation for $w, r$ and $D$. An action of an agent in some period $t$ is a vector $\left(c_{t}^{h}, m_{t}^{h}, n_{t}^{h}, k_{t+1}^{h}, \Delta_{t}^{h}\right) \in \mathbb{R}^{5}$, the action over the lifetime is $\sigma^{h}:=\left(c_{t}^{h}, m_{t}^{h}, n_{t}^{h}, k_{t+1}^{h}, \Delta_{t}^{h}\right)_{t \in T}$. The vector space which carries these actions is the countable infinite product of the vector space $\mathbb{R}^{5}$,

$$
Q^{h}:=\left(\mathbb{R}^{5}\right)^{\infty}
$$

The space $Q^{h}$ is endowed with the product topology. Let

$$
S:=\left(\mathbb{R}_{+}^{3}\right)^{\infty}
$$

\footnotetext{
${ }^{8}$ Note that the dividend which household $h \in H$ receives does not carry an index $h$. The rationale for this simplified notation is as follows: household $h \in H$ actually receives a dividend $D_{t}^{h}$ which is the mean value of the dividends $h$ gets from all firms $j \in J$ according to his shares $\theta_{j}^{h} \in[0,1]$. If $D_{t}^{j}$ is the profit of firm $j \in J$ in period $t$, then $D_{t}^{h}=\int_{J} \theta_{j}^{h} D_{t}^{j} d j$. However, since households are identical, $\theta_{j}^{h}=\theta_{j}$. The fact that shares sum up to one for every firm $j, \int_{H} \theta_{j} d h=1$, now implies $\int_{H} \theta_{j}^{h} d h=\theta_{j} \int_{0}^{1} d h=\theta_{j}=1$ for all $j \in J$. Since firms are symmetric, we know that $D_{t}^{j}=D_{t}$. It follows $D_{t}^{h}=\int_{J} \theta_{j}^{h} D_{t}^{j} d j=\int_{0}^{1} D_{t} d j=D_{t}$. This equality justifies the notation.
} 
be the space carrying price vectors of the form $\left(p_{t}, r_{t}, w_{t}\right)_{t \in T}$ and endow it with the product topology. Since the topological spaces $Q^{h}$ and $S$ endowed with the product topology are metrizable, we will treat them throughout as metric spaces.

The budget set of household $h$ is the correspondence $B^{h}: S \rightrightarrows Q^{h}$ defined by $^{9} B^{h}(p, r, w):=\left\{\sigma^{h}=(c, m, n, k, \Delta) \in Q^{h} \mid(1)-(5)\right\}$. The utility function of household $h$ is $U^{h}: Q^{h} \rightarrow \mathbb{R}$, defined by $\sigma \mapsto U^{h}(\sigma)$. His objective is to maximize this function by choosing a best element $\sigma^{h *} \in B^{h}(p, r, w)$. Note that the budget set is not convex due to the fixed transactions costs.

We will frequently argue in terms of real variables instead of nominal ones. For later reference let us therefore state the budget restrictions of an agent $h \in H$ in real terms before we continue. Denote the gross rate of deflation with $\pi_{t-1}:=\frac{p_{t-1}}{p_{t}}$ and let real variables (measured in the consumption good) be denoted with a tilde. Then the budget constraints read as

$$
\begin{aligned}
\tilde{m}_{t}^{h}+c_{t}^{h}+\kappa_{c}^{1}\left(\Delta_{t}^{h}\right) & \leq \tilde{\Delta}_{t}^{h}+\tilde{m}_{t-1}^{h} \pi_{t-1} \\
\tilde{n}_{t}^{h}+k_{t+1}^{2}+\tilde{\Delta}_{t}^{h}+\kappa_{c}^{2}\left(\Delta_{t}^{h}\right) & \leq w_{t}\left(e-\kappa_{l}\left(\Delta_{t}^{h}\right)\right)+k_{t}^{h}\left(r_{t}+1-\delta\right)+\tilde{n}_{t-1}^{h} \pi_{t-1}+D_{t} .
\end{aligned}
$$

\section{$2.3 \quad$ Firms}

Every firm $j$ demands labor $l_{t}^{j} \geq 0$ and rents capital $k_{t}^{j} \geq 0$ in competitive labor and capital markets. Denote firm $j$ 's output of the consumption good with $y_{t}^{j}$, the production function with $F(\cdot, \cdot)$ and the profit with $D_{t}^{j} \cdot j$ maximizes, in nominal terms, $p_{t} D_{t}^{j}:=p_{t} y_{t}^{j}-p_{t} r_{t} k_{t}^{j}-p_{t} w_{t} l_{t}^{j}$ in every period by choosing $l_{t}^{j}, k_{t}^{j}$ and $y_{t}^{j}$ under the restriction $y_{t}^{j} \leq F\left(l_{t}^{j}, k_{t}^{j}\right)$. In period $t$ the firms actions are summarized by $\left(y_{t}^{j}, l_{t}^{j}, k_{t}^{j}\right) \in \mathbb{R}_{+}^{3}$. An action vector summarizing the firm's action over all periods is denoted with $\sigma^{j}:=\left(y_{t}^{j}, l_{t}^{j}, k_{t}^{j}\right)_{t \in T}$. The vector $\sigma^{j}$ is an element of $\left(\mathbb{R}_{+}^{3}\right)^{\infty}$ endowed with the product topology. This space can also be treated as a metric space. Finally define $D^{j}:=\left(D_{t}^{j}\right)_{t}$.

\section{Competitive Equilibria}

Before we define a competitive equilibrium, we briefly describe the interaction of firms and households in this economy. To understand this interaction, one can imagine a bank in which all households $h \in H$ hold their checking account. Figure 1 illustrates the flow of real terms. The flow of the consumption good is from firms to households, the labor flow is in the opposite direction. Households accumulate the claims on capital as assets in the bank.

\footnotetext{
${ }^{9}$ For notational reasons, we suppress the parameters in the notation of the budget set.
} 


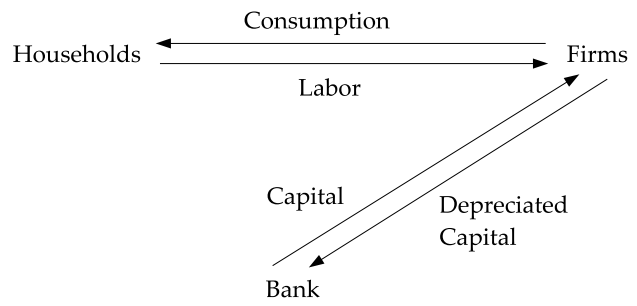

Figure 1: The interactions in this economy: The real side.

The claims on capital flow in this period into the firm sector allowing the firms to use the physical capital for production. Claims on capital from the previous period left over after depreciation flow in this sense back to the bank. Figure 2 illustrates the money flow in a period. Every arrow in the figure represents a particular flow of money. Firms pay wages and rents for capital in money on the checking accounts of households, i.e to the bank. Every household pays money for consumption reasons from the cash account to firms. Some households may choose to transfer some nominal wealth between his two accounts in this period. However, this need not be true for every household since they are free to hold money over time to save the transactions costs. ${ }^{10}$ This is why the set of households is disassembled in two subsets, one subset of households with transfers in this period and one without.

Define total transactions costs in the output good by $\kappa_{c}(\cdot):=\kappa_{c}^{1}(\cdot)+\kappa_{c}^{2}(\cdot)$. Consumption, labor, capital and money markets clear, if, $\forall t \in T$,

$$
\begin{aligned}
\int_{H}\left(c_{t}^{h}+k_{t+1}^{h}+\kappa_{c}\left(\Delta_{t}^{h}\right)\right) d h & =\int_{J} y_{t}^{j} d j+(1-\delta) \int_{H} k_{t}^{h} d h \\
\int_{H}\left(e-\kappa_{l}\left(\Delta_{t}^{h}\right)\right) d h & =\int_{J} l_{t}^{j} d j=: l_{t}^{d} \\
\int_{H} k_{t}^{h} d h & =\int_{J} k_{t}^{j} d j=: k_{t}^{d}, \\
\int_{H}\left(m_{t}^{h}+n_{t}^{h}\right) d h & =\int_{H}\left(m_{t-1}^{h}+n_{t-1}^{h}\right) d h .
\end{aligned}
$$

\footnotetext{
${ }^{10}$ One might wonder how this statement relates to the fact that households are identical. The nonconvexity of the budget sets, however, implies that there will typically be several best actions for the household in his budget set. This implies that households of the same type will be taking different actions among which they are indifferent.
} 


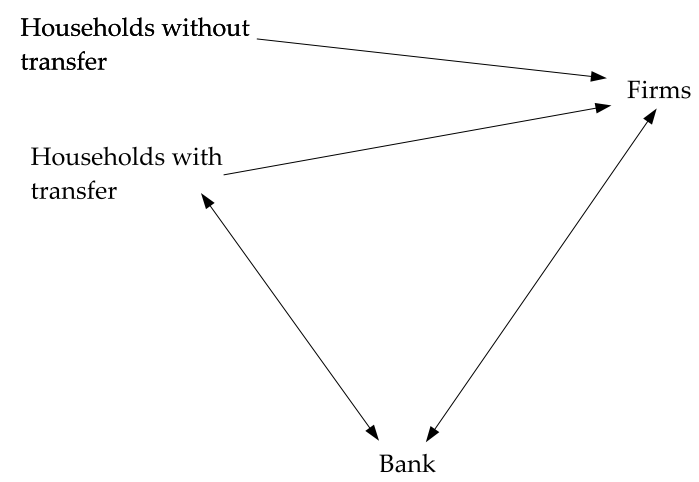

Figure 2: The interactions in this economy: The monetary side.

Denote aggregate supply with $\int_{J} y_{t}^{j} d j=: y_{t}$. Since $p_{t}$ is the price of the output good in terms of money, $p_{t}^{-1}$ is the price of fiat money in period $t$ measured in the output good.

Definition 1 A Competitive Equilibrium for this economy is a tuple $\left\{\left(\bar{\sigma}^{h}\right)_{h \in H},\left(\bar{\sigma}^{j}\right)_{j \in J}, \bar{p}, \bar{w}, \bar{r}\right\}$ such that

(i) markets clear at the vector $\left\{\left(\bar{\sigma}^{h}\right)_{h \in H},\left(\bar{\sigma}^{j}\right)_{j \in J}, \bar{p}, \bar{w}, \bar{r}\right\}$,

(ii) $\bar{\sigma}^{h}$ maximizes $U^{h}$ such that $\bar{\sigma}^{h} \in B^{h}(\bar{p}, \bar{w}, \bar{r})$ for almost every $h \in H$,

(iii) $\bar{\sigma}^{j}$ is a maximizer of $D^{j}$ given $(\bar{p}, \bar{w}, \bar{r})$ for almost every $j \in J$.

A Competitive Equilibrium is said to be monetary if the price of fiat money is positive in every period.

\section{Existence of Equilibrium}

In this Section, we formulate and explain the assumptions we impose to get existence of a monetary equilibrium. Denote with $F_{a}$ the partial derivative of $F$ with respect to argument $a$.

Assumption $1(i) \quad F$ is continuous and strictly increasing on $\mathbb{R}_{+}^{2}$, continuously differentiable on $\mathbb{R}_{++}^{2}$, has constant returns to scale and satisfies $F(l, 0)=0 ;($ ii $)$ For every $k>0, \lim _{l \rightarrow 0} F_{l}(l, k)=+\infty, \lim _{l \rightarrow+\infty} F_{l}(l, k)=0$ and 
for every $l>0, \lim _{k \rightarrow+\infty} F_{l}(l, k)=+\infty ;($ iii $)$ For every $l>0, \lim _{k \rightarrow 0} F_{k}(l, k)=$ $+\infty, 0 \leq \lim _{k \rightarrow+\infty} F_{k}(l, k)-\delta<\frac{1}{\beta}-1$.

Part (i) of this Assumption is a standard regularity assumption. Part (ii) imposes the standard Inada conditions for labor input. In addition, we explicitly require that the marginal product of labor diverges as capital input diverges. In economic terms, if the capital stock becomes infinite, then the wage becomes infinite, too. Part (iii) in the first place says that the Inada condition at zero capital input is true. However, we violate the typical assumption that the marginal product of capital converges to zero as capital runs out of bounds. Indeed, we assume that the marginal product of capital is bounded below by the rate of depreciation. The reason for this is as follows: In equilibrium, the rental rate equals the marginal product of capital. Hence, the marginal productivity of capital in this model is always large enough to make $r+1-\delta$ not smaller than one. In an equilibrium, households can then accumulate their labor income as real wealth on the checking account over time to afford a transfer in some finite future period. If the interest rate would be arbitrary close to zero, then the household could perhaps never be able to transfer some wealth from the checking account to the cash account. ${ }^{11}$ Finally, $\lim _{k \rightarrow+\infty} F_{k}(l, k)-\delta<\frac{1}{\beta}-1$ implies that for all large enough $k$ it holds that $\beta\left(F_{k}(l, k)+1-\delta\right)<1$. This property is required to argue that the aggregate capital stock remains finite in equilibrium.

Assumption 2 Households are identical with respect to their utility functions and endowments. Utilities are given by the function

$$
U^{h}\left(\sigma^{h}\right)=\sum_{t \in T} \beta^{t} u\left(c_{t}^{h}\right)
$$

where $\beta \in(0,1)$ is a discount factor and $u\left(c_{t}^{h}\right)=\ln c_{t}^{h}$.

The assumption that the one-period utility function is logarithmic is imposed to simplify some arguments in the proof of the theorem.

Assumption $3 \bar{m}^{h}>0$ and $\bar{k}^{h}>0$.

Assumption 4 Define the value $\bar{k}$ by the equation $\beta\left(F_{k}\left(e-\gamma_{l}, \bar{k}\right)+1-\delta\right)=$ 1. There exists a $\widetilde{k}>\bar{k}$ such that $\gamma_{c}=F\left(e-\gamma_{l}, \widetilde{k}\right)-\widetilde{k} \delta$.

\footnotetext{
${ }^{11}$ In fact, the series which only accumulates the wages in every period on the checking account could converge to a number which is smaller than the transactions costs in the output good.
} 
While Assumption 3 does not require comments, Assumption 4 is crucial and needs to be discussed: at the level of capital which would be the steady-state level in the frictionless economy using labor net of transactions costs, $\bar{k}$, the net output is strictly less than the transactions costs in output. This assumption is sufficient to avoid the constellation that every household wants to transfer wealth in every period. This could be consistent with an equilibrium in which money has no value at all.

Taking Assumptions (1) and (4) together, they imply that for every $k<\widetilde{k}$ we have $\gamma_{c}>F\left(e-\gamma_{l}, k\right)-k \delta$, and for every $k>\widetilde{k}$ we have $\gamma_{c}<F(e-$ $\left.\gamma_{l}, k\right)-k \delta$. Note that the assumption bounds the transactions costs in the output good below and above.

The next assumption is very unproblematic in that it just says that the transactions costs in labor must not exceed the endowment of labor:

Assumption $5 e>\gamma_{l}>0$.

It becomes clear that the assumptions we need in order to show the existence of an equilibrium in this Baumol-Tobin economy cannot be considered as innocent, in particular Assumptions 1 and 4. Perhaps one can interpret this fact by saying that the textbook theory of Baumol-Tobin is far too oversimplified and hence in this sense not very convincing. On the other hand, note that the set of assumptions is, as always, only sufficient, they might be quite far from being necessary. For some readers, this might be a counterargument to the accusation that the assumptions are strong.

Theorem Under Assumptions 1 - 5, there exists a Monetary Competitive Equilibrium.

The theorem is proven in the appendix. In the next section we remark on some properties of an equilibrium and interpret the structure of the model.

\section{$5 \quad$ Remarks}

Remark 1 In the following list, we summarize some properties of the equilibrium we show to exist. The proofs of these properties are included in the proof of the Theorem.

(i) There is a $R<+\infty$ and a $c>0$ such that $c<\pi_{t-1} \leq r_{t}+1-\delta<R$ for every $t \in T$. That is, gross deflation is uniformly bounded away from zero, the interest rate is uniformly bounded away from infinity 
and money is (weakly) dominated as a store of value on the checking account. $^{12}$

(ii) For (almost) every $h \in H$, there is a first $t<+\infty$ such that $\Delta_{t}^{h}>0$, and for every $s \in T$ with $\Delta_{s}^{h}>0$ there is a finite integer $\tau>0$ such that $\Delta_{s+\tau}^{h}>0$. That is, (almost) every household make a first transfer after finitely many periods and there is no last transfer.

(iii) There are $h \in H$ with positive measure having $\Delta_{t}^{h}=0$ for infinitely many $t \in T$.

(iv) The equilibrium capital stock remains finite.

Remark 2 We call this economy a Baumol-Tobin economy for the following reasons: First, money held on the cash account can be used for consumption at zero costs, where wealth from capital accumulation must be transferred at fixed costs to the cash account to finance consumption. This structure allows money to survive as a store of value since it has advantages in terms of marketability. Second, households actions show a transactions pattern which is similar to the original sawtooth pattern since households visit "the bank" from time to time but not everybody does so in every period (Parts (ii) and (iii) of Remark 1). Third, Assumption 4 implicitly defines a lower bound on the transactions costs. It is precisely this lower bound which eventually makes sure that money has a positive value in equilibrium. We will implicitly show that Assumption 4 is sufficient not to allow everybody to transfer wealth in every period. Importantly, this means that households hold money as a store of value on the cash account between two transaction dates. The only purpose for doing so is the transactions motive.

Remark 3 Romer (1986) called his model with a similar structure a monetary model with a "flexible Clower constraint" (Romer (1986), p. 666). He emphasizes that goods must be bought with money, but that the time period over which money is held is not exogenously fixed. In contrast, in a typical cash-in-advance economy the number of periods over which an agent holds money is by construction always one. Households receive their

\footnotetext{
${ }^{12}$ The intuition for this is very simple. Suppose that one has an equilibrium with positive consumption in which the real return on money is strictly larger than the return on capital in some period. Then, by a simple no-arbitrage argument, no household will hold capital on the checking account since it is dominated as a store of value. However, this implies that the capital stock in the next period will be zero. From this we know that the overall supply of the physical output good will be zero. This is not compatible with positive consumption in the equilibrium. Condition (i) is hence necessary for the existence of a non-trivial equilibrium.
} 
income in the evening of a day and need to hold it over night to use it either on the asset or on the commodity markets at the next day. The model presented in this paper can be interpreted similarly as the one in Romer (1986). The demand for money as a store of value is not derived by an exogenously imposed structure like the timing of markets. Instead, households money demand is determined endogenously by portfolio choice. Thus, households also choose the number of periods over which to hold the money endogenously. But note that they are not forced to hold the money over time at all.

Remark 4 The introduction of fixed costs which have to be paid if a transfer occurs implies that the households optimization problem is not convex. This complicates the analysis of such an economy drastically. Clearly, the Kuhn-Tucker conditions are not sufficient implying that the simplest existence argument in a macroeconomic model obviously fails. To get existence, we aggregate over a continuum of households to get the convexification of aggregate demand. Notice that it is the non-convex character of the transfer costs which makes households want to hold money over time to exploit the economies of scale which are inherent in this transactions technology.

\section{Concluding Remarks}

We conclude the paper by showing some directions of future research. In the first place, the analysis should be extended to allow for wage payments on the cash account. This can be done using a paycheck parameter which gives the fractions of the wages paid on both accounts. If all the wages are paid on the cash account, then the monetary aggregate which is modelled can be said to be broader. Indeed, it allows for the interpretation that the aggregate M1 can be held on the cash account, not only cash. As a consequence, the possibility to hold money also on the checking account is then not as meaningful as in the framework chosen here since this account should then be interpreted as an account for the interest bearing assets only. Second, the analysis can be extended to include a nominal bond as a second interest bearing asset in the economy. Households can incur some debt position over time since they can sell the bond short. This possibility should allow to get rid of the artificial restriction that the transactions costs must be paid on the account from which a transfer is financed, as formalized by the two separate mappings $\kappa^{1}$ and $\kappa^{2}$. As a third extension one can imagine a more general setting with several goods. Leaving the framework of the neoclassical model with one physical object complicates the analysis drastically since it is not 
clear how to formulate Assumption 4 in a more general economy. Finally, the analysis should be extended to allow for staggered wage payments. That is, one can assume that the wages are paid not every period but only from time to time. In the first place it can be analyzed how the interest rate process reacts to such a payment structure. If firms have to incur some debt to pay wages in advance for a whole month, say, then they will have to compete for the funds from the household sector at this date. One can suspect that the interest rates will tend to be higher at such points in time. As a consequence, optimal behavior should then imply that firms voluntarily choose heterogeneous wage payment dates over the month. Hence, the date of wage payments should then be included into the analysis (see Hellwig 1993).

\section{Appendix}

In this appendix we prove the theorem. The demand correspondence of household $h \in H$ is the mapping $\phi^{h}: S \rightrightarrows Q^{h}$ defined by

$$
\phi^{h}(p, r, w):=\underset{\sigma^{h} \in B^{h}(p, r, w)}{\arg \max } U^{h}\left(\sigma^{h}\right) .
$$

The extended demand correspondence $\bar{\phi}^{h}$ is the mapping $\bar{\phi}^{h}: S \rightrightarrows Q^{h} \times$ $\left(\mathbb{R}_{+}^{3}\right)^{\infty}$ defined by ${ }^{13}$

$$
\bar{\phi}^{h}(p, r, w):=\left\{\bar{\sigma}^{h}:=\left(\sigma^{h}, \kappa_{c}^{1}\left(\sigma^{h}\right), \kappa_{c}^{2}\left(\sigma^{h}\right), \kappa_{l}\left(\sigma^{h}\right)\right) \mid \sigma^{h} \in \phi^{h}(p, r, w)\right\} .
$$

Let us introduce the numbers $v, u \in \mathbb{N}$, and restrict prices $p$ to be in the set $P_{v u}:=\left\{p \in \mathbb{R}_{+}^{\infty} \mid \forall t \in T: p_{t} \in[1 / v, u]\right\}$, rental rates $r$ to be in the set $R_{v u}:=\left\{r \in \mathbb{R}_{+}^{\infty} \mid \forall t \in T: r_{t} \in\left[\delta+1 /(v u)^{2}, \delta+(v u)^{2}\right]\right\}$, and wages to be in the set $W_{v u}:=\left\{w \in \mathbb{R}_{+}^{\infty} \mid \forall t \in T: w_{t} \in[1 / v, v]\right\}$. The compact product space is denoted with

$$
S_{v u}:=P_{v u} \times R_{v u} \times W_{v u} .
$$

We treat all product spaces as metric spaces.

\footnotetext{
${ }^{13}$ The notation $\kappa_{j}\left(\sigma^{h}\right)$ is a shortcut for $\kappa_{j}^{h}\left(\Delta_{t}^{h}\right)$ where, for every $t, \Delta_{t}^{h}=\operatorname{proj}_{\Delta_{t}} \sigma^{h}$.
} 
Step 1 For every finite $v, u, B^{h}: S_{v u} \rightrightarrows Q^{h}$ is (i) nonempty, (ii) compactvalued and (iii) continuous.

Proof. (i) To see nonemptiness, just note that $0 \in B^{h}(p, r, w)$. (ii) To prove compactness of $B^{h}(p, r, w)$ for every finite $v, u$, it suffices to to show that every sequence in $B^{h}(p, r, w)$ has a subsequence which converges to a limit point in $B^{h}(p, r, w)$. Suppose to the contrary that there is a sequence $\sigma^{a} \rightarrow \sigma$ with $\sigma^{a} \in B^{h}(p, r, w)$ for all $a$, but $\sigma \notin B^{h}(p, r, w)$. For all $t \in T$, $\sigma^{a} \rightarrow \sigma$ implies $\sigma_{t}^{a} \rightarrow \sigma_{t}$. From the hypothesis $\sigma \notin B^{h}(p, r, w)$ it follows that there is a $t \in T$ such that at least one of the budget constraints is violated at $\sigma_{t}$. This strict inequality of one of the budget constraints implies that the violation of the budget must be true for all $a$ large enough. We now argue that this violation of the budget cannot be due to a jump in the fixed costs term. By definition of $\sigma_{t}, \Delta_{t}^{a} \rightarrow \Delta_{t}$ since $\sigma_{t}^{a} \rightarrow \sigma_{t}$. If $\Delta_{t} \neq 0$, then $\Delta_{t}^{a} \neq 0$ for all $a$ large enough so that the fixed costs cannot jump in the limit. If $\Delta_{t}=0$ but $\Delta_{t}^{a} \neq 0$ for all but finitely many $a$, then this jump will make the expenditure terms in the budget constraints jump downwards in the limit, but never upwards. Hence the hypothesis cannot be due to the fixed costs terms. It now follows from standard arguments that $\sigma \notin B^{h}(p, r, w)$ cannot be true if $\sigma^{a} \rightarrow \sigma$ with $\sigma^{a} \in B^{h}(p, r, w)$ for all $a$.

(iii) To see upper hemi-continuity of the budget set, let $\left(p^{a}, r^{a}, w^{a}\right) \rightarrow$ $(p, r, w), \sigma^{a} \in B^{h}\left(p^{a}, r^{a}, w^{a}\right)$ for all $a$ and $\sigma^{a} \rightarrow \sigma$. $\sigma \in B^{h}(p, r, w)$ follows from the same argument as given in part $(i i)$. Concerning lower hemicontinuity, let $\left(p^{a}, r^{a}, w^{a}\right) \rightarrow(p, r, w)$ and $\sigma \in B^{h}(p, r, w)$. We have to argue that there is a sequence $\sigma^{a} \rightarrow \sigma$ with $\sigma^{a} \in B^{h}\left(p^{a}, r^{a}, w^{a}\right)$ for all $a$ large enough. We make the argument conditional on the vector $\Delta$ which is one dimension of the limit action $\sigma$. Take an arbitrary $t \in T$. We will argue that there is a sequence of actions in $t$ converging to the limit action such that the budget constraints in $t$ are satisfied. Doing so, we take the action from period $t-1$ as given. Since this argument is applied for every $t \in T$, we get the desired result.

Suppose first that $\Delta_{t}<0$. Then we have in the limit $\frac{\left|\Delta_{t}\right|}{p_{t}} \leq \frac{m_{t-1}}{p_{t}}-$ $c_{t}-\frac{m_{t}}{p_{t}}-\gamma_{c}$ on the cash account. The hypothesis $\left|\Delta_{t}\right|>0$ implies that the right hand side of the former inequality is strictly positive. Since prices are finite, it is immediate that there are sequences $\Delta_{t}^{a} \rightarrow \Delta_{t}, c_{t}^{a} \rightarrow c_{t}$ and $m_{t}^{a} \rightarrow m_{t}$, where $\Delta_{t}^{a}<0$ for all $a$, such that $\frac{\left|\Delta_{t}^{a}\right|}{p_{t}^{a}} \leq \frac{m_{t-1}^{a}}{p_{t}^{a}}-c_{t}^{a}-\frac{m_{t}^{a}}{p_{t}^{a}}-\gamma_{c}$ for all $a$ large enough. Let $R_{t}:=r_{t}+1-\delta$. On the checking account, $k_{t+1}+\frac{n_{t}}{p_{t}} \leq$ $w_{t}\left(e-\gamma_{l}\right)+k_{t} R_{t}+\frac{\left|\Delta_{t}\right|}{p_{t}}=: y$, where $y>0$. Given the sequence $\Delta_{t}^{a} \rightarrow \Delta_{t}$ constructed to satisfy the cash account constraint, there are now sequences 
$k_{t+1}^{a} \rightarrow k_{t+1}$ and $n_{t}^{a} \rightarrow n_{t}$ satisfying $k_{t+1}^{a}+\frac{n_{t}^{a}}{p_{t}^{a}} \leq w_{t}^{a}\left(e-\gamma_{l}\right)+k_{t} R_{t}^{a}+\frac{\left|\Delta_{t}^{a}\right|}{p_{t}^{a}}$ for all large enough $a{ }^{14}$ That is all we have to show in this case.

Suppose now that $\Delta_{t}>0$ : We get for the checking account in the limit that $0<\frac{\Delta_{t}}{p_{t}} \leq w_{t}\left(e-\gamma_{l}\right)+k_{t} R_{t}+-\gamma_{c}-k_{t+1}-\frac{n_{t}-n_{t-1}}{p_{t}}=: y$. We can choose an appropriate sequence $\Delta_{t}^{a} \rightarrow \Delta_{t}$ with $\Delta_{t}^{a}>0$ for all $a$ and have to find sequences of actions such that $\frac{\Delta_{t}^{a}}{p_{t}^{a}} \leq w_{t}^{a}\left(e-\gamma_{l}\right)+k_{t} R_{t}^{a}-\gamma_{c}-k_{t+1}-\frac{n_{t}^{a}-n_{t}}{p_{t}^{a}}=: y^{a}$. Clearly, $y^{a} \rightarrow y$ and $y>0$, hence $y^{a}>0$ for all $a$ large enough. It follows that such a sequence exists for $a$ large enough. Given the sequence $\Delta_{t}^{a} \rightarrow \Delta_{t}$, the argument concerning the cash account is unproblematic and standard.

Finally, consider $\Delta_{t}=0$. In the limit we have on the checking account $k_{t+1}-\frac{n_{t}}{p_{t}} \leq w_{t} e+k_{t} R_{t}+\frac{n_{t-1}^{h}}{p_{t}}=: x$. Note that $x>0$ because $v$ finite implies that the wage is strictly positive. Choose the sequence $\Delta_{t}^{a}=0$ for all $a$. Define $w_{t}^{a} e+k_{t} R_{t}^{a}+\frac{n_{t-1}}{p_{t}^{a}}=: x^{a}$. This implies $x^{a} \rightarrow x$ and by observation $x>0$ implying $x^{a}>0$ for all $a$ large enough. Hence there are $k_{t+1}^{a} \rightarrow k_{t+1}$ and $n_{t}^{a} \rightarrow n_{t}$ satisfying $k_{t+1}^{a}+\frac{n_{t}^{a}}{p_{t}^{a}} \leq w_{t}^{a} e+k_{t} R_{t}^{a}+\frac{n_{t-1}}{p_{t}^{a}}$ for all $a$ large enough. The argument concerning the cash account is standard.

This finishes the proof for lower hemi-continuity and hence for continuity.

Step 2 For every finite $v, u$, it holds that $(i) \forall(p, r, w) \in S_{v u}, \phi^{h}(p, r, w) \neq$ $\emptyset$, (ii) $\phi^{h}$ is upper hemi-continuous and compact-valued on $S_{v u}$.

Proof. (i) Nonemptiness follows from the nonemptiness and the compactness of the budget set, as shown in the first part of the previous step, and the continuity of the utility function. (ii) Utility functions are continuous and, for all finite $v, u$, the budget sets have been shown to be continuous and compact-valued. The Maximum Theorem now immediately implies the upper hemi-continuity of the demand correspondence and the compactness of the demand sets on $S_{v u}$.

\footnotetext{
${ }^{14}$ Here we need that output transactions costs are defined by the separate mappings $\kappa^{1}$ and $\kappa^{2}$. If the household would be forced to pay the transactions costs also in the case $\Delta_{t}<0$ from the checking account, then the argument given in the text fails: instead of the number $y$ defined in the text, we would have $y-\gamma_{c}$. Nothing prevents us from supposing that this term is zero in the limit. Along the sequence, using the $\Delta_{t}^{a}$ which makes the cash account valid, the checking account could perhaps never be satisfied in certain circumstances. For suppose that $y^{a}-\gamma_{c}$ converges from below to zero, just because the prices sequence makes this happen. Since capital and money demand are nonnegative, there cannot exist such a sequence we are looking for.
} 
Step 3 For every finite $v, u$, it holds that $(i) \forall(p, r, w) \in S_{v u}, \bar{\phi}^{h}(p, r, w) \neq$ $\emptyset$, (ii) $\bar{\phi}^{h}$ is upper hemi-continuous and compact-valued on $S_{v u}$.

Proof. (i) The first statement follows immediately from what we established in the previous step. (ii) $\bar{\phi}^{h}$ is compact-valued on $S_{v u}$ since the budget set is compact-valued on $S_{v u}$. To see that the extended demand correspondence $\bar{\phi}^{h}$ is also upper hemi-continuous, take a sequence $\left(p^{a}, w^{a}, r^{a}\right) \rightarrow(p, w, r)$. In addition, let $\bar{\sigma}^{a} \in \bar{\phi}^{h}\left(p^{a}, w^{a}, r^{a}\right)$ and $\bar{\sigma}^{a} \rightarrow \bar{\sigma}$. We have to argue that $\bar{\sigma}^{h} \in \bar{\phi}^{h}(p, w, r)$. By the previous step and the definition of the extended demand, there is a sequence $\sigma^{a} \in \phi^{h}\left(p^{a}, w^{a}, r^{a}\right)$ with the property $\sigma^{a} \rightarrow \sigma \in \phi^{h}(p, w, r)$. Thus, it remains to show that the sequence of fixed costs generated from this action sequence converges, i.e. $\kappa\left(\sigma^{a}\right) \rightarrow \kappa(\sigma)$ for this sequence of $\sigma^{a}$, if $\kappa(\cdot)$ denotes the vector $\left(\kappa_{c}^{1}(\cdot), \kappa_{c}^{2}(\cdot), \kappa_{l}(\cdot)\right)$. Suppose this is not true. Then $\kappa(\cdot)$ jumps in the limit, but not before, i.e. $\kappa\left(\sigma^{a}\right)=\gamma$ for infinitely many $a$, but $\kappa(\sigma)=0$ (if $\sigma^{a}$ would be such that the limit $\Delta$ was non-zero, then it would be non-zero for every large enough $a$ and $\kappa(\sigma)$ would eventually converge). This contradicts optimality: since $\left(p^{a}, w^{a}, r^{a}\right) \rightarrow(p, w, r)$ and $\sigma^{a} \rightarrow \sigma^{h}$, the budget equations converges with $a$. By optimality, they must hold with equality along the sequence. But our hypothesis implies that we have a strict inequality in the limit.

Denote with $S_{t, v u}$ the projection of $S_{v u}$ onto $t$ and let $\sigma_{t}^{j}\left(p_{t}, w_{t}, r_{t}\right):=$ $\left(y^{j}\left(p_{t}, w_{t}, r_{t}\right), k^{j}\left(p_{t}, w_{t}, r_{t}\right), l^{j}\left(p_{t}, w_{t}, r_{t}\right)\right)$ be firm $j^{\prime}$ 's supply and demand on the output, capital and labor markets, respectively, which solve the maximization problem at prices $\left(p_{t}, w_{t}, r_{t}\right) \in S_{t, v u} .{ }^{15}$ The aggregate, which is by symmetry identical, is denoted by $\sigma_{t}^{J}\left(p_{t}, w_{t}, r_{t}\right):=\left(y\left(p_{t}, w_{t}, r_{t}\right), k^{d}\left(p_{t}, w_{t}, r_{t}\right)\right.$, $\left.l^{d}\left(p_{t}, w_{t}, r_{t}\right)\right)$. Finally, $\sigma^{J}(p, w, r):=\left\{\sigma_{t}^{J}\left(p_{t}, w_{t}, r_{t}\right)\right\}_{t \in T}$. Using Assumption 1 , standard arguments immediately imply the following result:

Step 4 For every finite $v, u, \sigma^{J}: S_{v u} \rightarrow\left(\mathbb{R}_{+}^{3}\right)^{\infty}$ is bounded and continuous.

For presentational reasons, let $Q^{M}:=\left(\mathbb{R}^{3}\right)^{\infty}$ be the space carrying the actions on the consumption, labor and capital markets in every period, respectively. Household's demand on these markets is a mapping $\Xi^{h}: S \rightrightarrows Q^{M}$

\footnotetext{
${ }^{15}$ The nominal price of the output good does not enter the objective of the firm. We include it in the demand correspondence to have a uniform notation.
} 
defined by

$$
\begin{array}{r}
\Xi^{h}(p, w, r):=\left\{\xi^{h}=\left(\xi_{t}^{h}\right)_{t \in T} \in Q^{M} \mid \forall t \in T: \xi_{t}^{h}:=\left(\xi_{c t}^{h}, \xi_{l t}^{h}, \xi_{k t}^{h}\right),\right. \text { where } \\
\xi_{c t}^{h}:=c_{t}^{h}+k_{t+1}^{h}+\kappa_{c}\left(\Delta_{t}^{h}\right)-k_{t}^{h}(1-\delta), \\
\left.\xi_{l t}^{h}:=-\left(e-\kappa_{l}\left(\Delta_{t}^{h}\right)\right), \xi_{k t}^{h}:=-k_{t}^{h}, \text { with } \bar{\sigma}^{h} \in \bar{\phi}^{h}(p, w, r)\right\} .
\end{array}
$$

Notice that we use the extended demand sets $\bar{\phi}^{h}(p, w, r)$ defined in (9). Define firms market demand by $\Xi^{J}: S \rightrightarrows Q^{M}$ by

$$
\begin{aligned}
\Xi^{J}(p, w, r):=\left\{\xi^{J}=\left(\xi_{t}^{J}\right)_{t \in T} \in Q^{M} \mid\right. & \forall t \in T: \xi_{t}^{J}:=\left(\xi_{c t}^{J}, \xi_{l t}^{J}, \xi_{k t}^{J}\right) \text { where } \\
& \xi_{c t}^{J}:=y_{t}, \xi_{l t}^{J}:=-l_{t}^{d}, \xi_{k t}^{J}:=-k_{h}^{d}, \\
& \text { with } \left.\left(y_{t}, k_{t}^{d}, l_{t}^{d}\right)_{t \in T}=\sigma^{J}(p, w, r)\right\} .
\end{aligned}
$$

Excess demand is the mapping $\Xi: S \rightrightarrows Q^{M}$ defined by

$$
\Xi(p, w, r):=\Xi^{h}(p, w, r)-\Xi^{J}(p, w, r)
$$

with generic element

$$
\xi=\left(\xi_{c t}, \xi_{l t}, \xi_{k t}\right)_{t \in T}=\left(\xi_{c t}^{h}-\xi_{c t}^{J}, \xi_{l t}^{h}-\xi_{l t}^{J}, \xi_{k t}^{h}-\xi_{k t}^{J}\right)_{t \in T} .
$$

Let $K_{v u} \subset Q^{M}$ be a compact and convex cube containing the set $\Xi\left(S_{v u}\right)$. The existence of such a set follows for all finite $v$ and $u$ from what we established before. $K_{t, v u}$ denotes the projection of $K_{v u}$ onto the $t$-th component, i.e. $K_{t, v u}=K_{c t, v u} \times K_{l t, v u} \times K_{k t, v u}$, where the latter sets are one-dimensional sets carrying the excess demands on consumption, labor and capital markets in $t$, respectively. By Tychonoff's Theorem, every coordinate set is a compact subset of $\mathbb{R}$. Define for every $t \in T$ the mapping $\zeta_{c t, v u}: K_{c t, v u} \rightrightarrows[1 / v, u]$ by

$$
\zeta_{c t, v u}\left(\xi_{c t, v u}\right):=\arg \max \left\{p_{t, v u} \xi_{c t, v u} \mid p_{t, v u} \in[1 / v, u]\right\},
$$

the mapping $\zeta_{l t, v u}: K_{l t, v u} \rightrightarrows[1 / v, v]$ by

$$
\zeta_{l t, v u}\left(\xi_{l t, v u}\right):=\arg \max \left\{w_{t, v u} \xi_{l t, v u} \mid w_{t, v u} \in[1 / v, v]\right\},
$$

and the mapping $\zeta_{k t, v u}: K_{k t, v u} \rightrightarrows\left[\delta+1 /(v u)^{2}, \delta+(v u)^{2}\right]$ by

$$
\zeta_{k t, v u}\left(\xi_{k t, v u}\right):=\arg \max \left\{r_{t, v u} \xi_{k t, v u} \mid r_{t, v u} \in\left[\delta+1 /(v u)^{2}, \delta+(v u)^{2}\right]\right\} .
$$


Next, define $\zeta_{t, v u}: K_{t, v u} \rightrightarrows[1 / v, u] \times[1 / v, v] \times\left[\delta+1 /(v u)^{2}, \delta+(v u)^{2}\right]$ by

$$
\zeta_{t, v u}:=\zeta_{c t, v u} \times \zeta_{l t, v u} \times \zeta_{k t, v u}
$$

and finally the product mapping $\zeta_{v u}: K_{v u} \rightrightarrows S_{v u}$ by

$$
\zeta_{v u}:=\prod_{t \in T} \zeta_{t, v u}
$$

Let $\overline{c o} \Xi^{h}$ denote the closure of the convex hull of $\Xi^{h}$ and define the correspondence

$$
\left(\overline{c o} \Xi^{h}-\Xi^{J}\right) \times \zeta_{v u}: K_{v u} \times S_{v u} \rightrightarrows K_{v u} \times S_{v u}
$$

by

$$
(\xi,(p, r, w)) \mapsto\left(\overline{c o} \Xi^{h}(p, r, w)-\Xi^{J}(p, r, w), \zeta_{v u}(\xi)\right) .
$$

Step 5 For every $(v, u)$, there is a fixed point

$$
\left(\xi_{v u}^{*},\left(p_{v u}^{*}, r_{v u}^{*}, w_{v u}^{*}\right)\right) \in\left(\overline{c o} \Xi^{h}\left(p_{v u}^{*}, r_{v u}^{*}, w_{v u}^{*}\right)-\Xi^{J}\left(p_{v u}^{*}, r_{v u}^{*}, w_{v u}^{*}\right), \zeta_{v u}\left(\xi_{v u}^{*}\right)\right) .
$$

Proof. We will show that the map $\left(\overline{c o} \Xi^{h}-\Xi^{J}\right) \times \zeta_{v u}: K_{v u} \times S_{v u} \rightrightarrows K_{v u} \times$ $S_{v u}$ is upper hemi-continuous, nonempty, compact and convex valued and that the set $K_{v u} \times S_{v u}$ is a nonempty, compact and convex subset of a locally convex Hausdorff space. The Kakutani-Fan-Glicksberg Fixed Point Theorem (Aliprantis, Border, 1999, Corollary 16.51) then implies the existence of a fixed point for every $v, u$.

$K_{v u} \times S_{v u}$ is locally convex since it is a metric space. It satisfies the Hausdorff Axiom since it is the countable product of Hausdorff spaces. (Folland (1999), Proposition 4.10). Convexity and nonemptiness of the mapping are obvious. $\zeta_{v u}$ and $\Xi^{J}$ are obviously upper hemi-continuous.

$\overline{c o} \Xi^{h}(p, r, w)$ is contained in a compact cube which is a subset of $Q^{M}$. Hence the set $\overline{c o} \Xi^{h}(p, r, w)$ is compact since closed subsets of compact sets are in turn compact. By construction, $\Xi^{h}$ is upper hemi-continuous since $\bar{\phi}^{h}(p, r, w)$ is upper hemi-continuous. The upper hemi-continuity of $\overline{c o} \Xi^{h}$ now follows from the upper hemi-continuity of $\Xi^{h}$ by using the result (Aliprantis, Border, 1999, Theorem 16.36) that the closed convex hull of an upper hemi-continuous correspondence is in turn upper hemi-continuous provided that the range of the closure of the convex hull of the correspondence is compact. 
Taking products and summing up upper hemi-continuous and compact valued correspondences preserves these properties (Aliprantis and Border, 1999, Theorems 16.28, 16.32). From this we infer that $\left(\overline{c o} \Xi^{h}-\Xi^{J}\right) \times \zeta_{v u}$ is upper hemi-continuous with compact values since $\overline{c o} \Xi^{h}, \Xi^{J}$ and $\zeta_{v u}$ have these properties.

We deal with a sequence of fixed points indexed by $v$ and $u$. We first hold $u$ fixed and let $v \rightarrow+\infty$. We can choose subsequences, denoted as the original sequence, which have a unique limit point as $v \rightarrow+\infty$. We denote such a limit by $\left(\xi_{u}^{*},\left(p_{u}^{*}, r_{u}^{*}, w_{u}^{*}\right)\right):=\lim _{v \rightarrow+\infty}\left(\xi_{v u}^{*},\left(p_{v u}^{*}, r_{v u}^{*}, w_{v u}^{*}\right)\right)$. Note that it is not clear a priori whether the limit of the subsequence is finite or not. From now on, we replace the original sequence with such a subsequence. In the next step, we show that the aggregate capital stock is bounded away from zero along every such subsequence.

Step 6 Define $k_{t, v u}^{*}$ to be aggregate capital supply in $t$ in the fixed point with number vu. There exists $a b>0$ such that for every $t$ and $u, \underset{v \rightarrow+\infty}{\lim } k_{t, v u}^{*}>b$.

Proof. Suppose the claim is wrong. Then, for every $b>0$, there is a $t(b)$ and a $u(b)$ such that $\lim _{v \rightarrow+\infty} k_{t(b), v u(b)}^{*} \leq b$. Choose a sequence $\left\{b^{n}\right\}_{n}$ with $b^{n}>0$ for all $n$ such that $\lim _{n \rightarrow+\infty} b^{n}=0$. There are corresponding sequences $\left\{t\left(b^{n}\right)\right\}_{n}$ and $\left\{u\left(b^{n}\right)\right\}_{n}$ which we denote with $\left\{t^{n}\right\}_{n}:=\left\{t\left(b^{n}\right)\right\}_{n}$ and $\left\{u^{n}\right\}_{n}:=\left\{u\left(b^{n}\right)\right\}_{n}$ for better readability. Taking subsequences, if necessary, we may assume that $\left\{b^{n}\right\}_{n},\left\{t^{n}\right\}_{n}$ and $\left\{u^{n}\right\}_{n}$ converge. It follows that $\lim _{n \rightarrow+\infty} \lim _{v \rightarrow+\infty} k_{t^{n}, v u^{n}}^{*}=0$. By the nonnegativity of capital, $\lim _{n \rightarrow+\infty v \rightarrow+\infty} \lim _{t^{n}, v u^{n}}^{h *}$ $=0$ for almost every $h \in H$.

Note first that for every $h \in H$ and for every finite $v$ and $u$, there is a sequence $\left\{t_{v u}^{h a}\right\}_{a \in \mathbb{N}} \subset T$, such that $\tilde{\Delta}_{t_{v u}^{h a}, v u}^{h *}>0$ and $t_{v u}^{h a} \rightarrow \infty$ as $a \rightarrow \infty$. This simply follows from the positive wage rate for $v$ finite and the fact that the real return on capital, $r_{t, v u}^{*}+1-\delta$, is always at least one. Hence households can accumulate real wealth on the checking account to afford a transfer after finitely many periods. This implies that the constraints on the checking account bind.

Taking subsequences, if necessary, we may assume that for every fixed $n$ and $u$ and for all large enough $v$ either $\pi_{t^{n}-1, v u^{n}}^{*}<r_{t^{n}, v u^{n}}^{*}+1-\delta$ or $\pi_{t^{n}-1, v u^{n}}^{*}>r_{t^{n}, v u^{n}}^{*}+1-\delta$ or $\pi_{t^{n}-1, v u^{n}}^{*}=r_{t^{n}, v u^{n}}^{*}+1-\delta$. In the rest of the proof, we will distinguish three scenarios according to these possibilities. We will mostly argue with fixed $n$ and hence implicitly assume that $n$ is large enough to make the respective argument work. 
Scenario 1: Suppose that, for some large enough $n$, there is a $v_{1}$ such that for all $v>v_{1}, \pi_{t^{n}-1, v u^{n}}^{*}<r_{t^{n}, v u^{n}}^{*}+1-\delta$. We can have three constellations:

Case (i): There exists a $v_{2}$ such that for all $v>v_{2}, k_{t^{n}, v u^{n}}^{d *}<k_{t^{n}, v u^{n}}^{*}$. Hence, as $v \rightarrow \infty, k_{t^{n}, v u^{n}}^{d *} \rightarrow k_{t^{n}, u^{n}}^{d *} \in\left[0, b^{n}\right]$ and, as $n \rightarrow \infty, k_{t^{n}, u^{n}}^{d *} \rightarrow 0$. In every fixed point, this is only consistent with $\frac{k_{t^{n}, u^{n}}^{d *}}{l_{t^{n}, u^{n}}} \rightarrow 0$ as $n \rightarrow \infty$. For if there is a subsequence such that $\frac{k_{t^{n}, u^{n}}^{d *}}{l_{t^{n}, u^{n}}^{d *}} \rightarrow x>0$ as $n \rightarrow \infty$, there must exist a positive sequence $\left\{L^{n}\right\}_{n}$ with $L_{n} \rightarrow 0$ as $n \rightarrow \infty$, such that $l_{t^{n}, u^{n}}^{d *} \leq L^{n}$. Hence, for every $n$, it holds that for every $\epsilon>0$, there is a $\bar{v}$ such that for all $v \geq \bar{v}, l_{t^{n}, v u^{n}}^{d *} \leq L^{n}+\epsilon$. Since $L_{n} \rightarrow 0$, there is a $N$ such that for all $n \geq N, 2 L^{n}<e-\gamma_{l}$. Choose $\epsilon=L^{n}$ and $n \geq N$ to get that for all $v \geq \bar{v}$, $l_{t^{n}, v u^{n}}^{d *} \leq L^{n}+\epsilon=2 L^{n}<e-\gamma_{l}$. Then it follows that for all $v \geq \bar{v}, w_{t^{n}, v u^{n}}=\frac{1}{v}$. For arbitrary $n$ and $v$, profit maximization and the linear homogeneity of the production function imply $w_{t^{n}, v u^{n}}=F_{l}\left(l_{t^{n}, v u^{n}}^{d *}, k_{t^{n}, v u^{n}}^{d *}\right)=F_{l}\left(1, \frac{k_{t^{n}, v u^{n}}^{d *}}{l_{t^{n}, v u^{n}}}\right)$. For every $n \geq N$, taking limits for $v \rightarrow \infty$ and using the continuity of the derivative implies $0=F_{l}\left(1, \frac{k_{t^{n}, u^{n}}^{d *}}{l_{t^{n}, u^{n}}^{d *}}\right)$. For every large enough $n$, Assumption 1 implies a contradiction since $\frac{k_{t^{n}, u^{n}}^{d *}}{l_{t^{n}, u^{n}}^{d *}}$ is arbitrary close to $x$, a positive number. Thus, $\frac{k_{t^{n}, u^{n}}^{d *}}{l_{t^{n}, u^{n}}^{d *}} \rightarrow 0$ as $n \rightarrow \infty$.

Profit maximization and the linear homogeneity of the production function imply $r_{t^{n}, v u^{n}}=F_{k}\left(1, \frac{k_{t^{n}, v u^{n}}^{d *}}{l_{t^{n}, v u^{n}}^{d *}}\right)$ or equivalently $\frac{k_{t^{n}, v u^{n}}^{d *}}{l_{t^{n}, v u^{n}}^{d *}}=F_{k}^{-1}\left(1, r_{t^{n}, v u^{n}}\right)$. For all $v>\max \left\{v_{1}, v_{2}\right\}$, we get from the excess supply on the capital market that $r_{t^{n}, v, u^{n}}^{*}=\delta+\frac{1}{\left(v u^{n}\right)^{2}}$. Taking limits as $v \rightarrow \infty, r_{t^{n}, u^{n}}^{*}=\delta$. However, from Assumption 1, $\infty=F_{k}^{-1}(1, \delta)$ which contradicts the fact that, for large enough $n, \frac{k_{t^{n}, v u^{n}}^{d *}}{l_{t^{n}, v u^{n}}^{d *}}$ is arbitrary close to zero.

Case (ii): There exists a $v_{2}^{\prime}$ such that for all $v>v_{2}^{\prime}, k_{t^{n}, v u^{n}}^{d *}=k_{t^{n}, v u^{n}}^{*}$. Hence, as $v \rightarrow \infty$, both $k_{t^{n}, v u^{n}}^{*} \rightarrow k_{t^{n}, u^{n}}^{*} \in\left[0, b^{n}\right]$ and $k_{t^{n}, v u^{n}}^{d *} \rightarrow k_{t^{n}, u^{n}}^{*} \in$ $\left[0, b^{n}\right]$. It follows $k_{t^{n}, u^{n}}^{d *} \rightarrow 0$ as $n \rightarrow \infty$. As argued in Case (i), this implies that $\frac{k_{t^{n}, u^{n}}^{d *}}{l_{t^{n}, u^{n}}^{d *}} \rightarrow 0$ as $n \rightarrow \infty$. In turn, $r_{t^{n}, u^{n}}=F_{k}\left(1, \frac{k_{t^{n}, u^{n}}^{d *}}{l_{t^{n}, u^{n}}^{d *}}\right)$ implies together with Assumption 1 that $r_{t^{n}, u^{n}}$ diverges with $n$.

In Case (ii), the sequence $k_{t^{n}, v u^{n}}^{*} r_{t^{n}, v u^{n}}^{*}$ must be bounded above. Indeed, for every fixed $n$, using Assumption 1, it follows from $k_{t^{n}, v u^{n}}^{d *} \rightarrow K^{n} \in\left[0, b^{n}\right]$ that there is a $Y^{n}>0$ such that $y_{t^{n}, v u^{n}}^{*} \rightarrow y_{t^{n}, u^{n}}^{*}$ with $y_{t^{n}, u^{n}}^{*} \in\left[0, Y^{n}\right]$. Since 
$b^{n} \rightarrow 0$ as $n \rightarrow \infty$, it follows that $Y^{n} \rightarrow 0$. It is now easy to see that, for $n$ sufficiently large, a sufficiently large $k_{t^{n}, v u^{n}}^{d *} r_{t^{n}, v u^{n}}^{*}$ would eventually contradict profit maximization, which is, however, an ingredient of every fixed point.

(ii.1) Suppose that $\lim _{n \rightarrow \infty v \rightarrow \infty} \lim _{t^{n}, v u^{n}}^{*} r_{t^{n}, v u^{n}}^{*}=0$. Hence, for every $\epsilon>0$, there exists a $N(=N(\epsilon))$ such that for all $n \geq N$, the following is true: for every $\eta>0$, there is a $V(=V(n, \eta))$ such that for all $v \geq V, k_{t^{n}, v u^{n}}^{*} r_{t^{n}, v u^{n}}^{*}<$ $\varepsilon+\eta$. The latter property must hence also be true for almost every $h \in H$.

Choose $\epsilon$ and $\eta$ small enough. By a now familiar argument, we can assume that $n$ is large enough, in particular $n \geq N$. Note that for all $v \geq v_{1}$ it holds that for every $h \in H, \tilde{n}_{t^{n}-1, v u^{n}}^{h *}=0$ because $\pi_{t^{n}-1, v u^{n}}^{*}<r_{t^{n}, v u^{n}}^{*}+1-\delta$.

(ii.1.a) Suppose that there is a $v_{3}$ such that for all $v>\max \left\{v_{1}, v_{2}^{\prime}, V, v_{3}\right\}$, $\tilde{\Delta}_{t^{n}-1, v u^{n}}^{h *}>0$ for some $H^{\prime} \subset H$ with positive measure. If some $h \in H^{\prime}$ chooses, for $v>\max \left\{v_{1}, v_{2}^{\prime}, V, v_{3}\right\}$ and $n$ large enough, $k_{t^{n}, v u^{n}}^{h^{\prime}}=\epsilon>0$ instead of letting $k_{t^{n}, v u^{n}}^{h *} \rightarrow K^{n}$ with $K^{n} \in\left[0, b^{n}\right]$ (where $b^{n}$ can be chosen arbitrary small for large enough $n$ ) and takes the $\epsilon$ from the positive $\tilde{\Delta}_{t^{n}-1, v u^{n}}^{h *}, h$ earns $\epsilon\left(r_{t^{n}, v u^{n}}^{*}+1-\delta\right)$ in $t^{n}$, which eventually exceeds the transactions costs in $t^{n}$. Hence for large enough $v$, the household can increase his consumption in $t^{n}$ large enough to compensate for a small consumption loss in $t^{n}-1$ due to the change in transfers $\tilde{\Delta}_{t^{n}-1, v u^{n}}^{h *}$ and savings. He must be better off after this deviation, implying that this case is not compatible with best responses.

(ii.1.b) Suppose that there is a $v_{3}^{\prime}$ such that for all $v>\max \left\{v_{1}, v_{2}^{\prime}, V, v_{3}^{\prime}\right\}$, $\tilde{\Delta}_{t^{n}-1, v u^{n}}^{h *}=0$ for almost every $h \in H$. We know that $\tilde{n}_{t^{n}-1, v u^{n}}^{h *}=0$ and that the budget constraint on the checking account binds. From this we get

$$
\begin{aligned}
k_{t^{n}, v u^{n}}^{h *}= & k_{t^{n}-1, v u^{n}}^{h *}\left(r_{t^{n}-1, v u}^{*}+1-\delta\right)+w_{t^{n}-1, v u^{n}}^{*}\left(e-\kappa_{l}\left(\Delta_{t^{n}-1, v u^{n}}^{h *}\right)\right) \\
& +\tilde{n}_{t^{n}-2, v u}^{h *} \pi_{t^{n}-2, v u} .
\end{aligned}
$$

Since $k_{t^{n}, v u^{n}}^{h *} \rightarrow k_{t^{n}, u^{n}}^{h *} \in\left[0, b^{n}\right]$ and $b^{n} \rightarrow 0$ as $n \rightarrow \infty$, we infer from (11) that the successive limit of $k_{t^{n}-1, v u^{n}}^{h *} r_{t^{n}-1, v u}^{*}$ is also zero. From $r_{t^{n}, v u^{n}}^{*}$ diverging with $n$, we know that there is a sequence $\left\{\bar{b}^{n}\right\} \geq 0, \bar{b}^{n} \rightarrow 0$ as $n \rightarrow \infty$, such that, as $v \rightarrow \infty, k_{t^{n}-1, v u^{n}}^{h_{*}^{*}} \rightarrow k_{t^{n}-1, u^{n}}^{h *} \in\left[0, \bar{b}^{n}\right]$, i.e. $k_{t^{n}-1, v u^{n}}^{h *}$ must eventually converge to zero as well. This argument is true for almost every $h \in H$. To summarize, we get $h$-almost surely that after taking successive limits, $k_{t^{n}-1, v u^{n}}^{h *}$ converges to zero, $r_{t^{n}-1, v u}^{*}$ diverges, but $k_{t^{n}-1, v u^{n}}^{h *} r_{t^{n}-1, v u}^{*}$ converges to zero. We can now proceed by going one period backwards where we know that, for all $h \in H$,

$$
\begin{aligned}
& k_{t^{n}-1, v u^{n}}^{h *}+\tilde{n}_{t^{n}-2, v u^{n}}^{h *}+\tilde{\Delta}_{t^{n}-2, v u^{n}}^{h *}+\kappa_{c}^{2}\left(\Delta_{t^{n}-2, v u^{n}}^{h *}\right)=k_{t^{n}-2, v u^{n}}^{h *} \\
& \quad \times\left(r_{t^{n}-2, v u}^{*}+1-\delta\right)+w_{t^{n}-2, v u^{n}}^{*}\left(e-\kappa_{l}\left(\Delta_{t^{n}-2, v u^{n}}^{h *}\right)+\tilde{n}_{t^{n}-3, v u}^{h *} \pi_{t^{n}-3, v u} .\right.
\end{aligned}
$$


We have to distinguish another three subcases here:

(1) First suppose that there is a $v_{4}$ such that for every $v>\max \left\{v_{1}, v_{2}, V, v_{3}^{\prime}, v_{4}\right\}$, $\tilde{\Delta}_{t^{n}-2, v u^{n}}^{h *}=0$ for almost every household. Then, for every $v>\max \left\{v_{1}, v_{2}, V, v_{3}^{\prime}, v_{4}\right\}$,

$$
\begin{aligned}
& k_{t^{n}-1, v u^{n}}^{h *}+\tilde{n}_{t^{n}-2, v u^{n}}^{h *}=k_{t^{n}-2, v u^{n}}^{h *}\left(r_{t^{n}-2, v u}^{*}+1-\delta\right) \\
& \quad+w_{t^{n}-2, v u^{n}}^{*}\left(e-\kappa_{l}\left(\Delta_{t^{n}-2, v u^{n}}^{h *}\right)+\tilde{n}_{t^{n}-3, v u}^{h *} \pi_{t^{n}-3, v u}\right.
\end{aligned}
$$

holds $h$-almost surely.

(1a) Assume $\limsup _{n \rightarrow \infty} \lim _{v \rightarrow \infty} \int_{H} \tilde{n}_{t^{n}-2, v u^{n}}^{h *} d h>0$. This implies that there is a $N^{\prime}$ such that for all $n \geq N^{\prime}$, there is a $V^{\prime}\left(=V^{\prime}(n)\right)$ such that for all $v \geq V^{\prime}, \int_{H} \tilde{n}_{t^{n}-2, v u^{n}}^{h *} d h>0$. Necessarily, for all $n \geq N^{\prime}$ and for all $v \geq V^{\prime}, r_{t^{n}-1, v u^{n}}^{*}+1-\delta=\pi_{t^{n}-2, v u}^{*}$. Thus, $\tilde{n}_{t^{n}-2, v u^{n}}^{h *} \pi_{t^{n}-2, v u}^{*}$ must diverge in $t^{n}-1$, i.e. $\liminf _{n \rightarrow \infty} \lim _{v \rightarrow \infty} \int_{H} \tilde{n}_{t^{n}-2, v u^{n}}^{h *} \pi_{t^{n}-2, v u}^{*} d h=$ $\infty$. Since income on the checking account is becoming arbitrary large, an argument given in Gale and Hellwig (1984) ${ }^{16}$ directly implies that mean consumption in $t^{n}-1$ is becoming large for large $n \geq N^{\prime}$ and $v \geq V^{\prime}$. But in $t^{n}-1$, total supply converges to zero, as seen before. Hence $p_{t^{n}-1, v u^{n}}^{*}=u^{n}$ which implies $\pi_{t^{n}-2, v u^{n}}^{*} \leq 1$, a contradiction.

(1b) Assume $\lim _{n \rightarrow \infty} \lim _{v \rightarrow \infty} \int_{H} \tilde{n}_{t^{n}-2, v u^{n}}^{h *} d h=0$ which implies that $\lim _{n \rightarrow \infty} \lim _{i \rightarrow \infty} \tilde{n}_{t^{n}-2, v u^{n}}^{h *}=0$ for almost every $h \in H$. Hence for every small $\epsilon>0$, there is a $N^{\prime \prime}$ such that for all $n \geq N^{\prime \prime}$, it is true that for every small $\eta>0$ there is a $V^{\prime \prime}$ such that for all $v \geq V^{\prime \prime}, \tilde{n}_{t^{n}-2, v u^{n}}^{h *} \leq \epsilon+\eta$. By (13), for almost every $h \in H$, the term $k_{t^{n}-2, v u^{n}}^{h *}\left(r_{t^{n}-2, v u}^{*}+1-\delta\right)+w_{t^{n}-2, v u^{n}}^{*}\left(e-\kappa_{l}\left(\Delta_{t^{n}-2, v u^{n}}^{h *}\right)\right)$ $+\tilde{n}_{t^{n}-3, v u}^{h *} \pi_{t^{n}-3, v u}$ has the same convergence behavior, i.e. the successive limit is zero. In particular, there is a positive sequence $\left\{W^{n}\right\}_{n}$ with $W^{n} \rightarrow 0$ such that $w_{t^{n}-2, v u^{n}}^{*} \rightarrow w_{t^{n}-2, u^{n}}^{*} \in\left[0, W^{n}\right]$. Taking limits of the equation $w_{t^{n}-2, v u^{n}}^{*}=F_{l}\left(1, \frac{k_{t^{n}-2, v u^{n}}^{d *}}{l_{t^{n}-2, v u^{n}}}\right) \mathrm{im}-$ plies $w_{t^{n}-2, u^{n}}^{*}=F_{l}\left(1, \frac{k_{t^{n}-2, u^{n}}^{d *}}{l_{t^{n}-2, u^{n}}^{d *}}\right)$ which implies $\frac{k_{t^{n}-2, u^{n}}^{d *}}{l_{t^{n}-2, u^{n}}^{d *}} \rightarrow 0$. This is only consistent with $k_{t^{n}-2, u^{n}}^{d *} \rightarrow 0$ for if $\frac{k_{t^{n}-2, u^{n}}^{d *}}{l_{t^{n}-2, u^{n}}^{d^{*}}} \rightarrow 0$ is

\footnotetext{
${ }^{16}$ See Footnote 9 in Gale and Hellwig (1984).
} 
induced by $l_{t^{n}-2, u^{n}}^{d *} \rightarrow \infty$ but $k_{t^{n}-2, u^{n}}^{d *} \nrightarrow 0$, we get excess demand on the labor market which implies an unbounded wage, a contradiction. $r_{t^{n}-2, u^{n}}^{*}=F_{k}\left(\frac{k_{t^{n}-2, u^{n}}^{d *}}{l_{t^{n}-2, u^{n}}^{d *}}, 1\right)$ implies in turn that $r_{t^{n}-2, u}^{*}$ diverges. Using (13), the successive limit of $k_{t^{n}-2, v u^{n}}^{h *}$ must be zero. Hence we can go one period backwards and start the same kind of arguments again. That is, this case is consistent at this point and we proceed with it below.

(2) Suppose next that, for some large enough $n$, there is a $v_{4}^{\prime}$ such that for every $v>\max \left\{v_{1}, v_{2}, V, v_{3}^{\prime}, v_{4}^{\prime}\right\}$, there is a $H^{\prime \prime} \subset H$ with positive measure such that for all $h \in H^{\prime \prime} \tilde{\Delta}_{t^{n}-2, v u^{n}}^{h *}>0$. This case eventually leads to a contradiction, as argued in (ii.1.a).

(3) Suppose finally that, for some large enough $n$, there is a $v_{4}^{\prime \prime}$ such that for every $v>\max \left\{v_{1}, v_{2}, V, v_{3}^{\prime}, v_{4}^{\prime \prime}\right\}$, there is a there is a $H^{\prime \prime \prime} \subset H$ with positive measure such that for all $h \in H^{\prime \prime \prime}, \tilde{\Delta}_{t^{n}-2, v u^{n}}^{h *}<0$. We only sketch the argument at this point: If on the one hand, for some large enough $n$ and for all large enough $v, r_{t^{n}-1, v u^{n}}^{*}+1-\delta>\pi_{t^{n}-2, v u^{n}}^{*}$, then money demand on the checking account is zero. But then capital supply in $t^{n}-1$ cannot become small, as argued in (ii.1.a), a contradiction. If on the other hand, the interest rate is equal to the deflation rate for some large enough $n$ and all large enough $v$, then $\tilde{n}_{t^{n}-2, v u}^{h *} \pi_{t^{n}-2, v u}^{*}$ must diverge. As argued above, consumption in $t^{n}-1$ will then be growing, while supply converges to zero, inducing $p_{t^{n}-1, v u^{n}}^{*}=u^{n}$ which implies $\pi_{t^{n}-2, v u^{n}}^{*} \leq 1$, a contradiction.

(ii.1.c) Suppose finally that there is a $v_{3}^{\prime \prime}$ such that for all $v>\max \left\{v_{1}, v_{2}, V, v_{3}^{\prime \prime}\right\}$, there is a $H^{\prime} \subset H$ with positive measure such that for all $h \in H^{\prime}, \tilde{\Delta}_{t^{n}-1, v u^{n}}^{h *}<0$. It is easy to see that the same arguments as above imply a contradiction.

Hence the only consistent case is subcase $(1 b)$ of subcase (ii.1.b). Repeating the argument eventually implies that, for almost every household, it holds that the successive limit of $k_{1, v u^{n}}^{h *}$ is zero, of $r_{1, v u^{n}}^{*}$ it is infinite, but of the term $k_{1, v u^{n}}^{h *} r_{1, v u^{n}}^{*}$ it is zero. However, every household is endowed with $k_{1}^{h}>0$ units of capital. It is not hard to see that supplying $k_{1, v u^{n}}^{h *}=k_{1}^{h}$ is the best response for a household given this constellation since the intertemporal utility approaches the supremum with this strategy. ${ }^{17}$ This is a contradiction.

\footnotetext{
${ }^{17}$ Again, the precise argument can be found in Footnote 9 in Gale and Hellwig (1984).
} 
(ii.2) Suppose now that $\limsup _{n \rightarrow \infty} \lim _{v \rightarrow \infty} k_{t^{n}, v u^{n}}^{*} r_{t^{n}, v u^{n}}^{*}>0$, a subsequence converges to a strictly positive and finite number. Then, for a large enough chosen $n$, there must be a $H^{\prime} \subset H$ such that for all $h \in H^{\prime}, k_{t^{n}, v u^{n}}^{h *}$ becomes at least as fast arbitrary small as $v \rightarrow \infty$ as $r_{t^{n}, v u^{n}}^{*}$ becomes large. In whatever subcase we are, the same arguments as above apply.

Case (iii): There is a $v_{3}^{\prime \prime}$ such that for all $v>v_{3}^{\prime \prime}$ we have $k_{t^{n}, v u^{n}}^{d *}>k_{t^{n}, v u^{n}}^{*}$. It suffices to sketch the proof here. By definition of $\zeta_{k t^{n}, v u^{n}}$, we get $r_{t^{n}, v u^{n}}^{*}=$ $\delta+\left(v u^{n}\right)^{2}$ for all large enough $v$. If deflation becomes large we get the same contradiction as before. If not, then the same arguments as above reveal that households maximization implies that $k_{t^{n}, v u^{n}}^{*} r_{t^{n}, v u^{n}}^{*}$ becomes large, hence $k_{t^{n}, v u^{n}}^{d *} r_{t^{n}, v u^{n}}^{*}$ becomes large even though $r_{t^{n}, v u^{n}}^{*}=\delta+\left(v u^{n}\right)^{2}$ implies $k_{t^{n}, v u^{n}}^{d *}$ becoming small. This obviously contradicts firms maximization.

Scenario 2: Suppose there is a $v_{2}^{\prime}$ such that for all $v>v_{2}^{\prime}, \pi_{t^{n}-1, v u^{n}}^{*}>$ $r_{t^{n}, v u^{n}}^{*}+1-\delta$ for some $n$. For all $v>v_{2}^{\prime}$, no household accumulates capital, $\int_{H} k_{t^{n}, v u^{n}}^{h *} d h=0$. Hence $\xi_{k t^{n}, v u^{n}}^{*}>0$ because capital demand is positive for finite interest rates, from what $r_{t^{n}, v u^{n}}^{*}=\delta+\left(v u^{n}\right)^{2}$. It follows from the hypothesis and our fixed point construction that $v u^{n} \geq \frac{p_{t^{n}-1, v u^{n}}^{*}}{p_{t^{n}, v u^{n}}^{*}}=$ $\pi_{t^{n}-1, v u^{n}}^{*}>r_{t^{n}, v u^{n}}^{*}+1-\delta=\delta+\left(v u^{n}\right)^{2}+1-\delta=\left(v u^{n}\right)^{2}+1$, from which $1>v u^{n}+\frac{1}{v u^{n}}$, a contradiction. ${ }^{18}$

Scenario 3: Suppose that, for some large enough $n$, there is a $v_{2}^{\prime \prime}$ such that for all $v>v_{2}^{\prime \prime}, \pi_{t^{n}-1, v u^{n}}^{*}=r_{t^{n}, v u^{n}}^{*}+1-\delta$.

(i) If, for all large enough $v$, we have $k_{t^{n}, v u^{n}}^{d *}<k_{t^{n}, v u^{n}}^{*}$, then the same argument as in Scenario 1 shows that this cannot be consistent. (ii) If for all large enough $v, k_{t^{n}, v u^{n}}^{d *}>k_{t^{n}, v u^{n}}^{*}$ then $r_{t^{n}, v u^{n}}^{*}=\delta+\left(v u^{n}\right)^{2}$. As in in the previous scenario, $v u \geq \frac{p_{t^{n}-1, v u^{n}}^{*}}{p_{t^{n}, v u}^{*}}=\pi_{t^{n}-1, v u^{n}}^{*}=r_{t^{n}, v u^{n}}^{*}+1-\delta=\left(v u^{n}\right)^{2}+1$, from which $1 \geq v u^{n}+\frac{1}{v u^{n}}$ a contradiction. (iii) If there is a $\tilde{v}_{3}$ such that for all $v>\tilde{v}_{3}$ we have $k_{t^{n}, v u^{n}}^{d *}=k_{t^{n}, v u^{n}}^{*}$, both terms converge with $v$ to a number in $\left[0, b^{n}\right]$ by the hypothesis of the step. As in Case (ii) of Scenario 1 we get that for all large enough $v, r_{t^{n}, v u^{n}}^{*}+1-\delta$ must diverge with $n$. For $v>\max \left\{v_{2}^{\prime \prime}, \tilde{v}_{3}\right\}, \pi_{t^{n}-1, v u^{n}}^{*}=r_{t^{n}, v u^{n}}^{*}+1-\delta$ implies then that $\pi_{t^{n}-1, v u^{n}}^{*}$ has the same convergence behavior. We continue with this case.

First, if for large enough $n$ there is a $\tilde{v}_{4}$ such that for all $v>\max \left\{v_{1}, v_{2}^{\prime \prime}, \tilde{v}_{3}, \tilde{v}_{4}\right\}$, there is a $H^{\prime} \subset H$ with positive measure such that

\footnotetext{
${ }^{18}$ This contradiction follows purely mechanically from the construction of the fixed point. It is possible to give another construction of the fixed point such that this contradiction arises from economic reasoning instead of pure mechanics. We use our construction just for simplicity.
} 
$\tilde{\Delta}_{t^{n}-1, v u^{n}}^{h *}>0$ for all $h \in H^{\prime}$, then $\pi_{t^{n}-1, v u^{n}}^{*} \tilde{m}_{t^{n}-1, v u^{n}}^{h *}$ must be unbounded for all $h \in H^{\prime}$ in $t^{n}$ implying again that $\int_{H} c_{t^{n}, v u^{n}}^{h *} d h$ is growing away from zero. By a now familiar argument, this implies $p_{t^{n}, v u^{n}}=u^{n}$ and thus $\pi_{t^{n}-1, v u^{n}}^{*} \leq 1$, a contradiction to the hypothesis that gross deflation is large.

Second, suppose there is a $\tilde{v}_{4}^{\prime}$ such that for all $v>\max \left\{v_{1}, v_{2}^{\prime \prime}, \tilde{v}_{3}^{\prime \prime}, \tilde{v}_{4}^{\prime}\right\}$ $\tilde{\Delta}_{t^{n}-1, v u^{n}}^{h *}=0$, for almost every $h \in H$. The same arguments as in $(i i .1 . b),(1 a)$ and $(1 b)$ apply.

Third, if there is a $v_{4}^{\prime \prime}$ such that for all $v>\max \left\{v_{1}, v_{2}^{\prime \prime}, \tilde{v}_{3}^{\prime \prime}, \tilde{v}_{4}^{\prime \prime}\right\}, \tilde{\Delta}_{t^{n}-1, v u^{n}}^{h *}<$ 0 for a positive measure of households, then $\int_{H} c_{t^{n}-1, v u^{n}}^{h *} d h$ must be bounded away from zero since agents forgo consumption. Without going into details, it is easy to see that it suffices in this case to consider $\tilde{m}_{t^{n}-1, v u^{n}}^{h *}$ being bounded away from zero for these agents for all $v>\max \left\{v_{1}, v_{2}^{\prime \prime}, \tilde{v}_{3}^{\prime \prime}, \tilde{v}_{4}^{\prime \prime}\right\}$. This implies $\pi_{t^{n}-1, v u^{n}}^{*} \tilde{m}_{t^{n}-1, v u^{n}}^{h *}$ running out of bounds and the same contradiction arises as before since a positive excess demand implies a deflation rate of at most one.

Step 7 For every $t, \pi_{t, v u}^{*} \leq r_{t+1, v u}^{*}+1-\delta$ for all $v$ and $u$.

Proof. This follows from the construction of the fixed point as argued in the second Scenario of the previous proof.

Step 8 For any given $t \in T$, the sequence $p_{t, v u}^{*}$ is bounded away from zero. In addition, for all $t \in T$ and for all $v$ large enough, $\xi_{c t, v u}^{*} \geq 0$.

Proof. Suppose there is a $t$ and a $u$ such that the sequence $p_{t, v u}^{*}$ converges to zero. By Step $7, \pi_{t-1, v u}^{*} \leq r_{t, v u}^{*}+1-\delta$ for all $v, u$ and $t$. There must be a finite $R$ which bounds $r_{t, v u}^{*}+1-\delta$ above for all $u$, all $t<+\infty$ and all large enough $v$. Indeed, we saw that $k_{t, v u}^{*}>b$ for some positive $b$, all $u$ and $t<+\infty$ and for all $v$ large enough. Hence, if such a $R$ does not exist and $r_{t, v u}^{*} \rightarrow+\infty$ with $v$ for some $t$ and $u$, we get excess supply on the capital market (capital demand goes to zero by Assumption 1) eventually implying $r_{t, v u}^{*}=\delta+1 /(v u)^{2}$, a contradiction from which we infer that the upper bound $R$ exists. Thus, $\pi_{t-1, v u}^{*}<R$ for all $t$. Since $p_{t, v u}^{*}$ becomes arbitrary small, we infer that $p_{t-1, v u}^{*}$ must also be very small since otherwise $\pi_{t-1, v u}^{*}$ becomes very large. By the same token $p_{1, v u}^{*}$ must be very small. Since $\bar{m}^{h}>0$, real wealth on the cash account in $t=1$ can be made arbitrary large. This eventually implies that consumption in period one is growing out of bounds ${ }^{19}$ and hence $\xi_{c 1, v u}^{*}>0$ - a contradiction since the price player

\footnotetext{
${ }^{19}$ The precise argument is exactly as given in Footnote 9 in Gale and Hellwig (1984).
} 
then chooses a price of $u$ in $t=1$. $\xi_{c t, v u}^{*} \geq 0$ for all $v$ large enough and for all $t$ follows now from the definition of $\zeta_{c t, v u}$.

Step 9 For every $t$ and $u, \lim _{v \rightarrow \infty} \xi_{l t, v u}^{*}=0$ and $\lim _{v \rightarrow \infty} \xi_{k t, v u}^{*}=0$.

Proof. Suppose there is a finite $t$ and $u$ with $\lim _{v \rightarrow \infty} \xi_{l t, v u}^{*}$ or $\lim _{v \rightarrow \infty} \xi_{l t, v u}^{*}$ being nonzero. We distinguish cases:

1. If both limits are strictly negative, then, as $v \rightarrow \infty, w_{t, v u}^{*} \rightarrow 0$ and $r_{t, v u}^{*} \rightarrow \delta$. The first order conditions are

$$
w_{t, v u}^{*}=F_{l}\left(\frac{l_{t, v u}^{d *}}{k_{t, v u}^{d *}}, 1\right)
$$

and

$$
r_{t, v u}^{*}=F_{l}\left(1, \frac{k_{t, v u}^{d *}}{l_{t, v u}^{d *}}\right)
$$

(14) implies together with Assumption 1 that $\frac{k_{t, v u}^{d *}}{l_{t, v u}^{d *}} \rightarrow 0$, but (15) implies together with Assumption 1 that $\frac{k_{t, v u}^{d *}}{l_{t, v u}^{d *}} \rightarrow \infty$, a contradiction to firms maximization.

2. If $\lim _{v \rightarrow \infty} \xi_{l t, v u}^{*}<0$ and $\lim _{v \rightarrow \infty} \xi_{k t, v u}^{*}>0$, then, as $v \rightarrow \infty, w_{t, v u}^{*} \rightarrow 0$ and $r_{t, v u}^{*} \rightarrow \infty$. (14) and (15) imply that $\frac{k_{t, v u}^{d *}}{l_{t, v u}^{d *}} \rightarrow 0$. If $k_{t, v u}^{d *} \rightarrow 0$, then for all large enough $v, \xi_{k t, v u}^{*}<0$ because capital supply is positive, a contradiction. If $l_{t, v u}^{d *} \rightarrow \infty$, then for all large enough $v, \xi_{k t, v u}^{*}>0$, a contradiction.

3. If $\lim _{v \rightarrow \infty} \xi_{l t, v u}^{*}<0$ and $\lim _{v \rightarrow \infty} \xi_{k t, v u}^{*}=0$, then, as $v \rightarrow \infty, w_{t, v u}^{*} \rightarrow 0$. (14) implies $\frac{k_{t, v u}^{d *}}{l_{t, v u}^{d *}} \rightarrow 0$. We claim that $l_{t, v u}^{d *} \rightarrow L_{t, u}^{d *}>e$. If not, then $k_{t, v u}^{d *} \rightarrow 0$, which contradicts $\lim _{v \rightarrow \infty} \xi_{k t, v u}^{*}=0$ since capital supply remains positive. Hence eventually $\xi_{l t, v u}^{*}>0$, a contradiction.

4. If both limits are positive, then, as $v \rightarrow \infty, w_{t, v u}^{*} \rightarrow \infty$ and $r_{t, v u}^{*} \rightarrow$ $\infty$. (14) implies together with Assumption 1 that $\frac{k_{t, v u}^{d *}}{l_{t, v u}^{d *}} \rightarrow \infty$, but (15) implies together with Assumption 1 that $\frac{k_{t, v u}^{d *}}{l_{t, v u}^{d *}} \rightarrow 0$, a contradiction to firms maximization.

5. If $\lim _{v \rightarrow \infty} \xi_{l t, v u}^{*}>0$ and $\lim _{v \rightarrow \infty} \xi_{k t, v u}^{*}<0$, then, as $v \rightarrow \infty, w_{t, v u}^{*} \rightarrow \infty$ and $r_{t, v u}^{*} \rightarrow \delta$. (14) and (15) imply that $\frac{k_{t, v u}^{d *}}{l_{t, v u}^{d *}} \rightarrow \infty$. Suppose that $k_{t, v u}^{d *} \rightarrow \infty$. 
As argued in Step 8, $p_{t, v u}^{*} \nrightarrow 0$ for all $t, r_{t, v u}^{*}$ is finite for all large enough $v$ and $\pi_{t, v u}^{*}$ is at most equal to $r_{t, v u}^{*}+1-\delta \leq R<+\infty$. Hence, all returns in the economy are finite. It follows easily that the households demand sets are contained in a compact cube. In particular, we get that capital supply is bounded above. Hence $\xi_{k t, v u}^{*}>0$ for large enough $v$, a contradiction. It follows that for all large enough $v, \xi_{k t, v u}^{*}>0$ which implies a contradiction. Suppose now that $l_{t, v u}^{d *} \rightarrow 0$. Then for all large enough $v, \xi_{l t, v u}^{*}<0$, a contradiction.

6. If $\lim _{v \rightarrow \infty} \xi_{l t, v u}^{*}>0$ and $\lim _{v \rightarrow \infty} \xi_{k t, v u}^{*}=0$, then, as $v \rightarrow \infty, w_{t, v u}^{*} \rightarrow \infty$. (14) implies $\frac{k_{t, v u}^{d *}}{l_{t, v u}^{d *}} \rightarrow \infty$. It follows $l_{t, v u}^{d *} \rightarrow 0$ for if not, then $k_{t, v u}^{d *} \rightarrow \infty$. Using the same arguments as in 5., it is easily seen that this contradicts the hypothesis $\lim _{v \rightarrow \infty} \xi_{k t, v u}^{*}=0$. However, $l_{t, v u}^{d *} \rightarrow 0$ contradicts $\lim _{v \rightarrow \infty} \xi_{l t, v u}^{*}>0$.

7. If $\lim _{v \rightarrow \infty} \xi_{k t, v u}^{*}<0$ and $\lim _{v \rightarrow \infty} \xi_{l t, v u}^{*}=0$, then, as $v \rightarrow \infty, r_{t, v u}^{*} \rightarrow \delta$. (15) implies $\frac{k_{t, v u}^{d *}}{l_{t, v u}^{d *}} \rightarrow \infty$. It follows $k_{t, v u}^{d *} \rightarrow \infty$ for if not, then $l_{t, v u}^{d *} \rightarrow 0$ which contradicts $\lim _{v \rightarrow \infty} \xi_{l t, v u}^{*}=0$. Using the same arguments as in $5 ., k_{t, v u}^{d *} \rightarrow \infty$ eventually implies $\xi_{k t, v u}^{*}>0$.

8. If $\lim _{v \rightarrow \infty} \xi_{k t, v u}^{*}>0$ and $\lim _{v \rightarrow \infty} \xi_{l t, v u}^{*}=0$, then, as $v \rightarrow \infty, r_{t, v u}^{*} \rightarrow \infty$. (15) implies $\frac{k_{t, v u}^{d *}}{l_{t, v u}^{d *}} \rightarrow 0$. It follows $k_{t, v u}^{d *} \rightarrow 0$ for if not, then $l_{t, v u}^{d *} \rightarrow \infty$ which contradicts $\lim _{v \rightarrow \infty} \xi_{l t, v u}^{*}=0$. A now familiar argument implies that $k_{t, v u}^{d *} \rightarrow 0$ is not consistent.

It follows that $\lim _{v \rightarrow \infty} \xi_{k t, v u}^{*}=\lim _{v \rightarrow \infty} \xi_{l t, v u}^{*}=0$ for all $t<+\infty$ and hence the same equations must hold in the limit $t \rightarrow+\infty$.

Step 10 Let $\lim _{v \rightarrow+\infty} k_{t, v u}^{*}=k_{t, u}^{*}$. Then $\lim _{t \rightarrow+\infty} k_{t, u}^{*}<+\infty$ for every $u$.

Proof. Suppose $k_{t, u}^{*}$ converges to infinity as $t \rightarrow+\infty$ for some finite $u$. Then there must necessarily be a $\iota \in H$ whose assets diverge, i.e. there is a $\iota \in H$ such that $k_{t+1, u}^{\iota *}+\tilde{n}_{t, u}^{\iota *}+\tilde{m}_{t, u}^{\iota *} \rightarrow+\infty$ as $t \rightarrow+\infty$. If this is not the case, then $\lim _{t \rightarrow+\infty} k_{t+1, u}^{h *} \leq \lim _{t \rightarrow+\infty}\left(k_{t+1, u}^{h *}+\tilde{n}_{t, u}^{h *}+\tilde{m}_{t, u}^{h *}\right) \leq+\infty$ for all $h \in H$ implying that aggregate capital cannot diverge. Since capital markets clear and $k_{t, u}^{*} \rightarrow+\infty$, it follows from Assumption 1 that there is a $\tilde{t}$ such that for all $t \geq \tilde{t}, \beta\left(r_{t, u}^{*}+1-\delta\right)<1$.

Choose some $\tau \geq \tilde{t}$ large enough to make sure that $k_{\tau+1, u}^{*}$ and $k_{\tau+1, u}^{\iota *}+$ $\tilde{n}_{\tau, u}^{\iota *}+\tilde{m}_{\tau, u}^{\iota *}$ are already large and such that agent $\iota$ chooses $\Delta_{\tau, u}^{\iota *} \neq 0$. We know that there is a finite $T$ such that $\Delta_{\tau+T, u}^{\iota *} \neq 0$ again. 
We can define a Kuhn-Tucker function for the maximization problem of every $h \in H$. The resulting Kuhn-Tucker conditions are necessary but not sufficient due to the lack of convexity. Since the transversality condition is derived from the necessary Kuhn-Tucker conditions, it is also necessary. We will argue that household $\iota$ eventually violates his transversality condition if $k_{t, u}^{*} \rightarrow+\infty$.

Let $\mu_{t, u}^{\iota, 1 *}$ be the (present value) Lagrange multiplier for the cash account constraint in period $t$ (formulated in real terms). Define (the current value multiplier) $\lambda_{t, u}^{\iota, 1 *}$ as usual by $\mu_{t, u}^{\iota, 1 *}=\lambda_{t, u}^{\iota, 1 *} \beta^{t}$. The multipliers for the checking account constraint in $t$ (also formulated in real terms) are denoted by $\mu_{t, u}^{\iota, 2 *}$ and $\lambda_{t, u}^{\iota, 2 *}$, where $\mu_{t, u}^{\iota, 2 *}=\lambda_{t, u}^{\iota, 2 *} \beta^{t}$. The transversality condition of agent $\iota \in H$ is then $\lim _{t \rightarrow+\infty}\left(\lambda_{t, u}^{\iota, 2 *} \beta^{t}\left(k_{t+1, u}^{\iota *}+\tilde{n}_{t, u}^{\iota *}\right)+\lambda_{t, u}^{\iota, 1 *} \beta^{t} \tilde{m}_{t, u}^{\iota *}\right)=0$.

For $\tau$ large enough, $\iota$ must accumulate his large investment between two successive transfer dates $\tau$ and $\tau+T$ either on the cash account or on the checking account. In the following Claim 1, we will argue that in case of large investments on the cash account, the whole term $\lambda_{t, u}^{\iota, 2 *} \beta^{t}\left(k_{t+1, u}^{\iota *}+\tilde{n}_{t, u}^{\iota *}\right)+$ $\lambda_{t, u}^{\iota, 1 *} \beta^{t} \tilde{m}_{t, u}^{\iota *}$ must eventually be strictly increasing in $t$ between such a pair of successive transfer dates $\tau$ and $\tau+T$. In Claim 2 we will show that in case of large accumulation of wealth on the checking account, at least $\lambda_{t, u}^{\iota, 2 *} \beta^{t}\left(k_{t+1, u}^{\iota *}+\tilde{n}_{t, u}^{\iota *}\right)$ must eventually be strictly increasing in $t$ between such a pair of successive transfer dates $\tau$ and $\tau+T$. Since the second term, $\lambda_{t, u}^{\iota, 1 *} \beta^{t} \tilde{m}_{t, u}^{\iota *}$, is nonnegative, the whole term, $\lambda_{t, u}^{\iota, 2 *} \beta^{t}\left(k_{t+1, u}^{\iota *}+\tilde{n}_{t, u}^{\iota *}\right)+$ $\lambda_{t, u}^{\iota, 1 *} \beta^{t} \tilde{m}_{t, u}^{\iota *}$, cannot converge to zero in this case as well. Since one out of these two cases is true between every pair of successive transfer dates, the transversality condition must be violated. This implies that the Step must be true.

Claim 1 Suppose that $\iota$ chooses to allocate his large investment between $\tau$ and $\tau+T$ on the cash account, and invests only relatively less on the checking account. Then, for every large enough $\tau, \lambda_{t, u}^{\iota, 2 *} \beta^{t}\left(k_{t+1, u}^{\iota *}+\tilde{n}_{t, u}^{\iota *}\right)+\lambda_{t, u}^{\iota, 1 *} \beta^{t} \tilde{m}_{t, u}^{\iota *}$ is increasing in $t$ between $\tau$ and $\tau+T$.

Proof. We first show that $\lambda_{\tau+j, u}^{\iota, 1 *} \beta^{\tau+j}=\lambda_{\tau+j, u}^{\iota, 2 *} \beta^{\tau+j}$ for all $j \in\{0, \ldots, T\}: \iota$ chooses to allocate most of his large investments on the cash account between $\tau$ and $\tau+T$. Hence $\tilde{m}_{\tau+j}^{\iota *}$ must be positive for all $j \in\{0, \ldots, T-1\}$. This can only be a best response if $\prod_{j=0}^{T-1} \pi_{\tau+j, u}^{*} \geq \prod_{j=0}^{T-1}\left(r_{\tau+j+1, u}^{*}+1-\delta\right)$. Since $\pi_{t-1, u}^{*} \leq r_{t, u}^{*}+1-\delta$, we get $\prod_{j=0}^{T-1} \pi_{\tau+j, u}^{*}=\prod_{j=0}^{T-1}\left(r_{\tau+j+1, u}^{*}+1-\delta\right)$. Clearly, $\pi_{\tau+j, u}^{*}=r_{\tau+j+1, u}^{*}+1-\delta$ for all $j \in\{0, \ldots, T-1\}$. Indeed, if $\pi_{\tau+j, u}^{*}<$ 
$r_{\tau+j+1, u}^{*}+1-\delta$ for some $j$ then $\prod_{j=0}^{T-1} \pi_{\tau+j, u}^{*}=\prod_{j=0}^{T-1}\left(r_{\tau+j+1, u}^{*}+1-\delta\right)$ implies that there must be another $j^{\prime}$ with $\pi_{\tau+j^{\prime}, u}^{*}>r_{\tau+j^{\prime}+1, u}^{*}+1-\delta$, a contradiction.

Since $\tilde{m}_{\tau+j}^{\iota *}$ is strictly positive for all $j \in\{0, \ldots, T-1\}$, the first order conditions imply $\frac{\lambda_{\tau+j+1, u}^{\iota, 1 *} \beta^{\tau+j+1}}{\lambda_{\tau+j, u}^{\iota, 1 *} \beta^{\tau+j}}=\frac{1}{\pi_{\tau+j, u}^{*}}$ for all $j \in\{0, \ldots, T-1\}$. On the checking account, optimality implies that $\iota$ must accumulate his wage income between $\tau+1$ and $\tau+T$. Hence, the investment $k_{\tau+1+j, u}^{\iota *}+\tilde{n}_{\tau+j, u}^{\iota *}$ in a "composite asset" having return $\max \left\{\pi_{t-1, u}^{*}, r_{t, u}^{*}+1-\delta\right\}=r_{t, u}^{*}+1-\delta$ must be strictly positive in these periods implying $\frac{\lambda_{\tau+j+1, u}^{\iota, 2 *} \beta^{\tau+j+1}}{\lambda_{\tau+j, u}^{\iota, 2 *} \beta^{\tau+j}}=\frac{1}{r_{\tau+j+1, u}^{*}+1-\delta}$ for all $j \in\{1, \ldots, T-1\}$. Furthermore, $\frac{\lambda_{\tau+1, u}^{\iota, 2 *} \beta^{\tau+1}}{\lambda_{\tau, u}^{L, 2 *} \beta^{\tau}} \leq \frac{1}{r_{\tau+1, u}^{*}+1-\delta}$.

Since $\Delta_{\tau, u}^{\iota *} \neq 0$ and $\Delta_{\tau+T, u}^{\iota *} \neq 0$, it follows from the Kuhn-Tucker conditions that $\lambda_{\tau, u}^{\iota, 2 *}=\lambda_{\tau, u}^{\iota, 1 *}$ and $\lambda_{\tau+T, u}^{\iota, 2 *}=\lambda_{\tau+T, u}^{\iota, 1 *}$.

We get $\frac{\lambda_{\tau+1, u}^{\iota, 2 *} \beta^{\tau+1}}{\lambda_{\tau, u}^{l, 2 *} \beta^{\tau}} \leq \frac{1}{r_{\tau+1, u}^{*}+1-\delta}=\frac{1}{\pi_{\tau, u}^{*}}=\frac{\lambda_{\tau+1, u}^{\iota, 1 *} \beta^{\tau+1}}{\lambda_{\tau, u}^{l, 1 *} \beta^{\tau}}=\frac{\lambda_{\tau+1, u}^{\iota, 1 *} \beta^{\tau+1}}{\lambda_{\tau, u}^{L, 2 *} \beta^{\tau}}$ from which we infer $\lambda_{\tau+1, u}^{\iota, 2 *} \beta^{\tau+1} \leq \lambda_{\tau+1, u}^{\iota, 1 *} \beta^{\tau+1}$. In the next period, $\frac{\lambda_{\tau+2, u}^{\iota, 2 *} \beta^{\tau+2}}{\lambda_{\tau+1, u}^{\iota, 2 *} \beta^{\tau+1}}=$ $\frac{1}{r_{\tau+2, u}^{*}+1-\delta}=\frac{1}{\pi_{\tau+1, u}^{*}}=\frac{\lambda_{\tau+2, u}^{\iota, 1 *} \beta^{\tau+2}}{\lambda_{\tau+1, u}^{\iota, 1 *} \beta^{\tau+1}}$. The previous inequality and this equality together imply $\lambda_{\tau+2, u}^{\iota, 2 *} \beta^{\tau+1} \leq \lambda_{\tau+2, u}^{\iota, 1 *} \beta^{\tau+1}$. The same argument can be made until period $\tau+T$ to find $\lambda_{\tau+T, u}^{\iota, 2 *} \beta^{\tau+1} \leq \lambda_{\tau+T, u}^{\iota, 1 *} \beta^{\tau+1}$. That is, if there is one strict inequality involved in some period between $\tau+1$ and $\tau+T-1$, we get the strict inequality in $\tau+T$. However, we know that $\lambda_{\tau+T, u}^{\iota, 2 *}=\lambda_{\tau+T, u}^{\iota, 1 *}$, so every weak inequality must hold as equality. This is what had to be shown.

The next step is to show that $\lambda_{t, u}^{\iota, 2 *} \beta^{t}\left(k_{t+1, u}^{\iota *}+\tilde{n}_{t, u}^{\iota *}\right)+\lambda_{t, u}^{\iota, 1 *} \beta^{t} \tilde{m}_{t, u}^{\iota *}$ is eventually increasing in $t$. From what we said before, the latter term reduces to $\lambda_{t, u}^{\iota, 2 *} \beta^{t}\left(k_{t+1, u}^{\iota *}+\tilde{n}_{t, u}^{\iota *}+\tilde{m}_{t, u}^{\iota *}\right)$. From the budget constraints $(6)$ and $(7)$ and from the equality of deflation and returns on capital, we get $k_{t+1, u}^{\iota *}+\tilde{n}_{t, u}^{\iota *}+$ $\tilde{m}_{t, u}^{\iota *}=\left(\tilde{m}_{t-1, u}^{\iota *}+\tilde{n}_{t-1, u}^{\iota *}\right) \pi_{t-1, u}^{*}+k_{t, u}^{\iota}\left(r_{t, u}^{*}+1-\delta\right)+w_{t, u}^{*}\left(e-\kappa_{l}\left(\Delta_{t, u}^{\iota}\right)\right)-$ $\kappa_{c}\left(\Delta_{t, u}^{\iota}\right)-c_{t, u}^{\iota}=\left(k_{t, u}^{\iota *}+\tilde{n}_{t-1, u}^{\iota *}+\tilde{m}_{t-1, u}^{\iota *}\right)\left(r_{t, u}^{*}+1-\delta\right)+w_{t, u}^{*}\left(e-\kappa_{l}\left(\Delta_{t, u}^{\iota}\right)\right)-$ $\left.\kappa_{c}\left(\Delta_{t, u}^{\iota}\right)\right)-c_{t, u}^{\iota}$. Hence

$$
\begin{aligned}
& \frac{\lambda_{t, u}^{\iota, 2 *} \beta^{t}}{\lambda_{t-1, u}^{\iota, 2 *} \beta^{t-1}} \frac{k_{t+1, u}^{\iota *}+\tilde{n}_{t, u}^{\iota *}+\tilde{m}_{t, u}^{\iota *}}{k_{t, u}^{\iota *}+\tilde{n}_{t-1, u}^{\iota *}+\tilde{m}_{t-1, u}^{\iota *}} \\
& \quad=\frac{1}{r_{t, u}^{*}+1-\delta}\left(r_{t, u}^{*}+1-\delta+\frac{\left.w_{t, u}^{*}\left(e-\kappa_{l}\left(\Delta_{t, u}^{\iota}\right)\right)-\kappa_{c}\left(\Delta_{t, u}^{\iota}\right)\right)-c_{t, u}^{\iota}}{k_{t, u}^{\iota *}+\tilde{n}_{t-1, u}^{\iota *}+\tilde{m}_{t-1, u}^{\iota *}}\right) .
\end{aligned}
$$


This term is strictly larger than one if and only if $w_{t, u}^{*}\left(e-\kappa_{l}\left(\Delta_{t, u}^{\iota}\right)\right)>$ $\left.\kappa_{c}\left(\Delta_{t, u}^{\iota}\right)\right)+c_{t, u}^{\iota}$. In the first place, consumption is decreasing in time since $\beta\left(r_{t, u}+1-\delta\right)<1$. Secondly, for a large enough capital stock, Assumption 1 implies that the wage rate becomes arbitrary large. Hence the fraction given above is larger than one for all large enough $t$, thus proving Claim 1.

Claim 2 Suppose that $\iota$ chooses to allocate his large investment between $\tau$ and $\tau+T$ on the checking account. Then, for every large enough $\tau$, $\lambda_{t, u}^{\iota, 2 *} \beta^{t}\left(k_{t+1, u}^{\iota *}+\tilde{n}_{t, u}^{\iota *}\right)$ is increasing in $t$ between $\tau$ and $\tau+T$.

Proof. Since $\iota$ invests on the checking account, the investment $k_{t+1+j, u}+$ $\tilde{n}_{t+j, u}^{\iota *}$ in the "composite asset" which has return $\max \left\{\pi_{t-1, u}^{*}, r_{t, u}^{*}+1-\delta\right\}=$ $r_{t, u}^{*}+1-\delta$ must be strictly positive between $\tau$ and $\tau+T$, i.e. $\frac{\lambda_{\tau+j+1, u}^{\iota, 2 *} \beta^{\tau+j+1}}{\lambda_{\tau+j, u}^{L, 2 *} \beta^{\tau+j}}=$ $\frac{1}{r_{\tau+j+1, u}^{*}+1-\delta}$ for all $j \in\{0, \ldots, T-1\}$. Using this fact together with the budget constraint allows us to derive the following fraction:

$$
\begin{aligned}
& \frac{\lambda_{t, u}^{\iota, 2 *} \beta^{t}}{\lambda_{t-1, u}^{\iota, 2 *} \beta^{t-1}} \frac{k_{t+1, u}^{\iota *}+\tilde{n}_{t, u}^{\iota *}}{k_{t, u}^{\iota *}+\tilde{n}_{t-1, u}^{\iota *}} \\
& \quad=\frac{1}{r_{t, u}^{*}+1-\delta}\left(r_{t, u}^{*}+1-\delta+\frac{w_{t, u}^{*}\left(e-\kappa_{l}\left(\Delta_{t, u}^{\iota *}\right)\right)-\kappa_{c}^{2}\left(\Delta_{t, u}^{\iota *}\right)-\tilde{\Delta}_{t, u}^{\iota *}}{k_{t, u}^{\iota *}+\tilde{n}_{t-1, u}^{\iota *}}\right) .
\end{aligned}
$$

This term is larger than one if and only if $w_{t, u}^{*}\left(e-\kappa_{l}\left(\Delta_{t, u}^{\iota *}\right)\right)>\kappa_{c}^{2}\left(\Delta_{t, u}^{\iota *}\right)+\tilde{\Delta}_{t, u}^{\iota *}$. This is obviously true for $t \in\{\tau+1, \ldots, \tau+T-1\}$ because, by definition of $\tau$ and $\tau+T, \kappa_{c}^{2}\left(\Delta_{t, u}^{\iota *}\right)+\tilde{\Delta}_{t, u}^{\iota *}=0$ in these periods. For a transfer date $s$, say, this is true if and only if $w_{s, u}^{*}\left(e-\gamma_{l}\right)>\gamma_{c}+\tilde{\Delta}_{s, u}^{\iota *}$. We will now argue that this will be true for all large enough $s$.

Optimization of $\iota$ and the fact that deflation rates are positive implies that $\iota$ finances positive consumption between $\tau$ and $\tau+T-1$ by holding money, hence $\tilde{m}_{\tau+j, u}^{\iota *}>0$ for all $j \in\{0, \ldots, T-2\}$. From this, $\frac{\lambda_{\tau+j+1, u}^{\iota, 1 *} \beta^{\tau+j+1}}{\lambda_{\tau+j, u}^{\iota, 1 *} \beta^{\tau+j}}=\frac{1}{\pi_{\tau+j, u}^{*}}$ for all $j \in\{0, \ldots, T-2\}$. We can restrict attention to the case where $\tilde{m}_{\tau+T-1, u}^{\iota *}=0$. Indeed, if this term would be strictly positive, then $\iota$ must be indifferent between holding wealth between $\tau$ and $\tau+T$ on the checking account and on the cash account. This can only be true if the rates of return between these two transfer dates coincide. But then we are back in the case of Claim 1.

For transfer date $\tau$, one calculates the transfer using the cash account constraints as $\tilde{\Delta}_{\tau, u}^{\iota *}=-\tilde{m}_{\tau-1}^{\iota *} \pi_{\tau-1, u}^{*}+\sum_{j=0}^{T-1} \frac{c_{\tau+j, u}^{\iota *}}{\pi_{\tau, u}^{*} \cdots \cdots \pi_{\tau+j-1, u}^{*}}$. The first order 
condition $\frac{\lambda_{\tau+j+1, u}^{\iota, 1 *} \beta^{\tau+j+1}}{\lambda_{\tau+j, u}^{\iota, 1 *} \beta^{\tau+j}}=\frac{1}{\pi_{\tau+j, u}^{*}}$ reduces to $\frac{c_{\tau+j, u}^{\iota *} \beta}{c_{\tau+j+1, u}^{\iota *}}=\frac{1}{\pi_{\tau+j, u}^{*}}$ using Assumption 2 which imposes log-utilities. Plugging this into the term for the transfer gives us the simple expression $\tilde{\Delta}_{\tau, u}^{\iota *}=-\tilde{m}_{\tau-1}^{\iota *} \pi_{\tau-1, u}^{*}+c_{\tau, u}^{\iota *} \sum_{j=0}^{T-1} \beta^{j}$. According to the reasoning given above, it follows that $\frac{\lambda_{t, u}^{\iota, 2 *} \beta^{t}}{\lambda_{t-1, u}^{L, 2 *} \beta^{t-1}} \frac{k_{t+1, u}^{\iota *}+\tilde{n}_{t, u}^{\iota *}}{k_{t, u}^{\iota *}+\tilde{n}_{t-1, u}^{\iota *}}$ is larger than one at a transfer date $\tau$ if and only if $w_{\tau, u}^{*}\left(e-\gamma_{l}\right)>\gamma_{c}-\tilde{m}_{\tau-1}^{\iota *} \pi_{\tau-1, u}^{*}+$ $c_{\tau, u}^{\iota *} \sum_{j=0}^{T-1} \beta^{j}+\gamma_{c}$. Since aggregate capital is diverging with $t$ and the wage is determined by the marginal product of labor using arbitrary large capital, Assumption 1 implies that the term $w_{\tau, u}^{*}\left(e-\gamma_{l}\right)$ is becoming arbitrary large if we choose $\tau$ large enough. The term $\sum_{j=0}^{T-1} \beta^{j}$ is bounded above by $\frac{1}{1-\beta}$ and $c_{t, u}^{\iota *}$ is strictly decreasing in $t$ implying that $c_{\tau, u}^{\iota *}$ becomes small for $\tau$ large enough. Hence, the above inequality condition must eventually be true implying that $\frac{\lambda_{t, u}^{L, 2 *} \beta^{t}}{\lambda_{t-1, u}^{\iota, 2 *} \beta^{t-1}} \frac{k_{t+1, u}^{\iota *}+\tilde{n}_{t, u}^{\iota *}}{k_{t, u}^{\iota *}+\tilde{n}_{t-1, u}^{\iota *}}>1$ for all large enough transfer dates $t$. This proves the Claim.

As argued before Claim 1, both Claims together imply that $\iota \in H$ must violate his transversality condition. But this contradicts optimality and thus proves the Step.

Define $s_{u}:=\min \left\{t \in T \mid p_{t u}=u\right\}$. If there is a finite $\bar{u}$ such that $s_{\bar{u}}=$ $+\infty$, then the vector $\left\{\left(\sigma_{\bar{u}}^{h *}\right)_{h \in H},\left(\sigma_{\bar{u}}^{j *}\right)_{j \in J}, p_{\bar{u}}^{*}, w_{\bar{u}}^{*}, r_{\bar{u}}^{*}\right\}$ is an equilibrium: all monetary prices are bounded above by $\bar{u}$, agents maximize (see the last step) and all markets clear. Indeed, it only remains to argue that the output and money markets clear since capital and labor markets have already been shown to clear. If some consumption market in some $t<+\infty$ would be in excess demand at this $\bar{u}$, i.e. $\xi_{c t, \bar{u}}^{*}>0$, then the price would hit the bound $\bar{u}$ in this $t$, a contradiction to $s_{\bar{u}}=+\infty$. Since excess supply is already excluded, the claim follows. Money market clearing follows from Walras law in every period $t \in T$ since prices are both positive and finite. Thus it remains to show that such an upper bound exists. The idea to prove existence by this argument is adopted from Gale and Hellwig (1984).

Step 11 There is a $u^{*}<+\infty$ such that $s_{u^{*}}=+\infty$.

Proof. The proof is by contradiction. So suppose from now on that $s_{u}<+\infty$ for all $u \in \mathbb{N}$. Note that for every $u \in \mathbb{N}$, markets clear in all periods $t<s_{u}$ by the same rationale as given a few lines above. 
Let $\bar{\phi}^{r e a l, h}(\pi, r, w)$ be the demand sets derived from $\bar{\phi}^{h}(p, r, w)$ by using the real budget sets instead of the nominal ones. ${ }^{20}$ The projection of these real demands onto real money demand on the cash account and on the checking account in a period $t$ are denoted with $\bar{\phi}_{\tilde{m}_{t}^{*}}^{r e a l, h}(\pi, r, w)$ and $\bar{\phi}_{\tilde{n}_{t}^{*}}^{\text {real,h}}(\pi, r, w)$, respectively.

Claim 3 There exist numbers $\bar{u}<+\infty, T<+\infty$ and $c>0$ with the property that for all $u>\bar{u}$, there is a subset of households $\bar{H} \subset H$ such that $t^{21}$

$$
\inf \left\{\int_{\bar{H}}\left(\bar{\phi}_{\tilde{m}_{s_{u}+j, u}}^{r e a l, h}\left(\pi_{u}^{*}, r_{u}^{*}, w_{u}^{*}\right)+\bar{\phi}_{\tilde{n}_{s_{u}+j, u}}^{r e a l, h}\left(\pi_{u}^{*}, r_{u}^{*}, w_{u}^{*}\right)\right) d h\right\}>c
$$

for some $0 \leq j<T$.

Proof. Suppose that the claim is false. Then, for every finite $\bar{u}$, finite $T$ and positive $c$, there is a $u>\bar{u}$ such that for all $0 \leq j<T$ and for all subsets $\bar{H} \subset H$ we have $\inf \left\{\int_{\bar{H}}\left(\bar{\phi}_{\tilde{m}_{s_{u}+j, u}}^{r e a l, h}\left(\pi_{u}^{*}, r_{u}^{*}, w_{u}^{*}\right)+\bar{\phi}_{\tilde{n}_{s_{u}+j, u}}^{r e a l, h}\left(\pi_{u}^{*}, r_{u}^{*}, w_{u}^{*}\right)\right) d h\right\}$ $\leq c$ for all $j \geq 0 . H \subset H$ implies that $\inf \left\{\int_{H}\left(\bar{\phi}_{\tilde{m}_{s_{u}+j, u}}^{r e a l, h}\left(\pi_{u}^{*}, r_{u}^{*}, w_{u}^{*}\right)+\right.\right.$ $\left.\left.\bar{\phi}_{\tilde{n}_{s_{u}+j, u}}^{r e a l, h}\left(\pi_{u}^{*}, r_{u}^{*}, w_{u}^{*}\right)\right) d h\right\} \leq c$. Since this is true for every finite $T$ and every positive $c$, we can choose a sequence of $T$ and $c$ converging to $+\infty$ and zero, respectively, to get that for every $j \geq 0, \inf \left\{\int_{H}\left(\bar{\phi}_{\tilde{m}_{s_{u}+j, u}}^{r e a l, h}\left(\pi_{u}^{*}, r_{u}^{*}, w_{u}^{*}\right)\right.\right.$ $\left.\left.+\bar{\phi}_{\tilde{n}_{s_{u}+j, u}}^{\text {real } h}\left(\pi_{u}^{*}, r_{u}^{*}, w_{u}^{*}\right)\right) d h\right\}=0$. Call this Hypothesis $(*)$.

We now argue that under $(*), \tilde{\Delta}_{t, u}^{h *}>0$ for almost all $h \in H$ and for all $t \geq s_{u}+1$. Indeed, suppose the number of periods between two transfers is positive for a positive measure of agents. Since real wages are bounded away from zero and interest rates are at least $\delta$, the number of such periods is always finite. The deflation factor is strictly positive since $0<p_{t, u}^{*} \leq u<+\infty$ for every $u<+\infty$. Hence between the two transfer dates, positive real money demand is budgetary feasible and allows for a positive consumption stream. The property $\ln (0)=-\infty$ imposed by Assumption 2 now implies that, if optimal behavior satisfies $(*)$, then, $\forall t \geq s_{u}+1, \tilde{\Delta}_{t, u}^{h *}>0$ must eventually be true for almost every $h$. The reason is that positive consumption can be financed by deviating to positive real money demand which allows to get an intertemporal utility which does not converge to $-\infty$.

Thus, under $(*)$, aggregate transactions costs in all $t \geq s_{u}+1$ are $\int_{H} \kappa_{c}^{2}\left(\Delta_{t, u}^{h *}\right) d h=\gamma_{c}$ and $\int_{H} \kappa_{l}\left(\Delta_{t, u}^{h *}\right) d h=\gamma_{l}$. Since the labor markets clear,

\footnotetext{
${ }^{20}$ Note that these two demand sets are one-to-one as long as the output prices are both strictly positive and finite.

${ }^{21}$ Note that the integral used in the condition is an integral over a correspondence.
} 
in every such period, the labor input is just $e-\gamma_{l}$. Households are homogeneous in all $t \geq s_{u}+1$. Remember that we denote aggregate variables by dropping the superscript.

Case (i): Suppose $k_{s_{u}+1, u}^{*}<\bar{k}$, the market clearing aggregate capital stock in $s_{u}+1$ is strictly smaller than $\bar{k}$. Integrate the checking accounts (7) (in real terms) over $h$ and use the fact that every $h$ sacrifices the transactions costs to get

$$
\begin{gathered}
c_{s_{u}+1, u}^{*}+k_{s_{u}+2, u}^{*}+\gamma_{c}=w_{s_{u}+1, u}^{*}\left(e-\gamma_{l}\right)+k_{s_{u}+1, u}^{*}\left(r_{s_{u}+1, u}^{*}+1-\delta\right) \\
+D_{s_{u}+1, u}^{*}-\left[\left(\tilde{m}_{s_{u}+1, u}^{*}+\tilde{n}_{s_{u}+1, u}^{*}\right)-\pi_{s_{u}, u}^{*}\left(\tilde{m}_{s_{u}, u}^{*}+\tilde{n}_{s_{u}, u}^{*}\right)\right]
\end{gathered}
$$

Define, $\forall t \in T$,

$$
\xi_{\tilde{m} t, u}^{*}:=\tilde{m}_{t, u}^{*}+\tilde{n}_{t, u}^{*}-\pi_{t-1, u}^{*}\left(\tilde{m}_{t-1, u}^{*}+\tilde{n}_{t-1, u}^{*}\right) .
$$

Use this definition and substitute for $D_{s+1, u}^{*}$ to get

$$
\begin{aligned}
& k_{s_{u}+2, u}^{*}-k_{s_{u}+1, u}^{*}=\left[y_{s_{u}+1}^{*}-k_{s_{u}+1, u}^{*} \delta-\gamma_{c}\right]-c_{s_{u}+1, u}^{*} \\
& -\left[w_{s_{u}+1, u}^{*} \xi_{l s+1, u}^{*}+r_{s_{u}+1, u}^{*} \xi_{k s_{u}+1, u}^{*}+\xi_{\tilde{m} s_{u}+1, u}^{*}\right] .
\end{aligned}
$$

By Hypothesis $(*)$ and the fact that labor and capital markets clear, the second brackets on the right hand side are zero. Since the labor market clears, every firm must be using the labor input $e-\gamma_{l}$. It follows that, $\forall j \in J$, $y_{s_{u}+1}^{*}=y_{s_{u}+1}^{j *}=F\left(l_{s_{u}+1, u}^{j *}, k_{s_{u}+1, u}^{j *}\right)=F\left(l_{s_{u}+1, u}^{d *}, k_{s_{u}+1, u}^{d *}\right)=F\left(e-\gamma_{l}, k_{s_{u}+1, u}^{*}\right)$ and thus

$$
k_{s_{u}+2, u}^{*}-k_{s_{u}+1, u}^{*}=\left[F\left(e-\gamma_{l}, k_{s_{u}+1, u}^{*}\right)-k_{s_{u}+1, u}^{*} \delta-\gamma_{c}\right]-c_{s_{u}+1, u}^{*} .
$$

$k_{s_{u}+1, u}^{*}<\bar{k}$ and the fact that firms play identical actions implies that $k_{s_{u}+1, u}^{j *}<\bar{k}$ for every $j \in J$. Assumption 4 now implies that the brackets on the right hand side of (17) are strictly negative. It follows

$$
c_{s_{u}+1, u}^{*}+k_{s_{u}+2, u}^{*}<k_{s_{u}+1, u}^{*}
$$

and hence

$$
k_{s_{u}+2, u}^{*}<k_{s_{u}+1, u}^{*},
$$

i.e., the aggregate capital stock shrinks between $s_{u}+1$ and $s_{u}+2$. Since under Hypothesis $(*), \tilde{\Delta}_{t, u}^{h *}>0$ for all $h \in H$ and for all $t \geq s_{u}+1$, households act homogeneously from period $s_{u}+1$ on. This implies that the following Euler equation must be met:

$$
\frac{u^{\prime}\left(c_{s_{u}, u}^{*}\right)}{u^{\prime}\left(c_{s_{u}+1, u}^{*}\right)}=\beta \cdot \max \left\{r_{s_{u}+1, u}^{*}+1-\delta, \pi_{s_{u}, u}^{*}\right\} .
$$


Suppose $r_{s_{u}+1, u}^{*}+1-\delta>\pi_{s_{u}, u}^{*}$. We know that $k_{s_{u}+1, u}^{d *}<\bar{k}$. By definition of $\bar{k}$, this implies $\frac{u^{\prime}\left(c_{s_{u}, u}^{*}\right)}{u^{\prime}\left(c_{s_{u}+1, u}^{*}\right)}=\beta\left(r_{s_{u}+1, u}^{*}+1-\delta\right)>1$, consumption grows between $s_{u}$ and $s_{u}+1$. If $\left(r_{s_{u}+1, u}^{*}+1-\delta\right)=\pi_{s_{u}, u}^{*}$, the same reasoning applies.

From (19), $r_{s_{u}+2, u}^{*}>r_{s_{u}+1, u}^{*}$. It follows that $\beta\left(r_{s_{u}+2, u}^{*}+1-\delta\right)>$ $\beta\left(r_{s_{u}+1, u}^{*}+1-\delta\right)>1$ and the same argument as above shows that consumption also grows between $s_{u}+1$ and $s_{u}+2$. In addition, integrating the constraints (in real terms) and rearranging as before gives

$$
\begin{aligned}
& k_{s_{u}+3, u}^{*}-k_{s_{u}+2, u}^{*}=\left[y_{s_{u}+2}^{*}-k_{s_{u}+2, u}^{*} \delta-\gamma_{c}\right]-c_{s_{u}+2, u}^{*} \\
& -\left[w_{s_{u}+2, u}^{*} \xi_{l s_{u}+2, u}^{*}+r_{s_{u}+2, \imath}^{*} \xi_{k s_{u}+2, u}^{*}+\xi_{\tilde{m} s_{u}+2, u}^{*}\right] .
\end{aligned}
$$

From $r_{s_{u}+2, u}^{*}>r_{s_{u}+1, u}^{*}>\delta$ we infer as before that firms have symmetric single valued demands and supplies. Hence, (19) and Assumption 4 again imply

$$
k_{s_{u}+3, u}^{*}<k_{s_{u}+2, u}^{*} .
$$

The same procedure shows that consumption grows over time for all $t \geq s_{u}+1$ and capital shrinks over time for all $t \geq s_{u}+1$. Similarly, one can show that (18) holds for all successor periods of $s_{u}+1$, i.e.

$$
c_{s_{u}+1+j, u}^{*}+k_{s_{u}+2+j, u}^{*}<k_{s_{u}+1+j, u}^{*}
$$

holds for every integer $j \geq 0$. Sum these inequalities over the next $T^{\prime}$ periods to get

$$
\sum_{j=0}^{T^{\prime}} c_{s_{u}+1+j, u}^{*}+k_{s_{u}+2+T^{\prime}, u}^{*}<k_{s_{u}+1, u}^{*} .
$$

Since $\sum_{j=0}^{T^{\prime}} c_{s_{u}+1+j, u}^{*}$ becomes with $T^{\prime}$ large enough, this is a contradiction.

If $k_{s_{u}+1}^{*} \in[\bar{k}, \widetilde{k}]$, then it is not hard to see that the same argument applies to this case. Hence, the aggregate capital stock decreases in time, and we eventually end up in Case (i) again.

Case (ii): Suppose that $k_{s_{u}+1}^{*}>\widetilde{k}$. As before, we know that the following two equations are true:

$$
\begin{aligned}
c_{s_{u}+1, u}^{*}+k_{s_{u}+2, u}^{*} & =F\left(e-\gamma_{l}, k_{s_{u}+1, u}^{*}\right)+(1-\delta) k_{s_{u}+1, u}^{*}-\gamma_{c} . \\
\frac{u^{\prime}\left(c_{s_{u}, u}^{*}\right)}{u^{\prime}\left(c_{s_{u}+1, u}^{*}\right)} & =\beta\left(F_{k}\left(e-\gamma_{l}, k_{s_{u}+1, u}^{*}\right)+1-\delta\right) .
\end{aligned}
$$


We now borrow an argument from the analysis of global dynamics in the neoclassical growth model. The previous two equations characterize the global dynamics of aggregate consumption and capital in this case. We start by deriving the two functions which indicate constant consumption and constant capital: First, the value $\bar{k}$ was defined such that

$$
1=\beta\left(F_{k}\left(e-\gamma_{l}, \bar{k}\right)+1-\delta\right) .
$$

At $\bar{k}$, consumption is unchanged for every $h$ since

$$
\frac{u^{\prime}\left(c_{t}\right)}{u^{\prime}\left(c_{t+1}\right)}=\beta\left(F_{k}\left(e-\gamma_{l}, \bar{k}\right)+1-\delta\right)=1
$$

In Figure 3, which is a $(c, k)$-diagram, the set of consumption-capital-vectors having this property is the vertical line at the value $\bar{k}$. Second, define $c(k)$ by

$$
c(k):=F\left(e-\gamma_{l}, k\right)-\delta k-\gamma_{c} .
$$

Aggregate capital remains constant if the aggregate consumption is equal to $c(k)$. From Assumptions 1 and 4, we easily infer that this function is negative for $k<\widetilde{k}$, zero at $\widetilde{k}$, positive for $k>\widetilde{k}$, has positive slope for all $k$ and the slope converges to a number in $\left[0, \frac{1}{\beta}-1\right)$ as $k$ runs out of bounds. This function is also indicated in Figure 3. In this figure, we further define the two areas $A$ and $B$ which are both located to the right of $\widetilde{k}$. $A$ lies above $c(k)$ and $B$ below. We draw the dashed line to indicate that the analysis of Case (ii) is concerned with the dynamics for $k>\widetilde{k}$ only. We will consider separately the two cases where $\left(k_{s_{u}+1}^{*}, c_{s_{u}+1, u}^{*}\right)$ lies in $A$ and in $B$.

Suppose first that the tuple $\left(k_{s_{u}+1}^{*}, c_{s_{u}+1, u}^{*}\right)$ is in area $B$. By definition, $c_{s_{u}+1, u}^{*}<c\left(k_{s_{u}+1}\right)$. Thus

$$
\begin{aligned}
k_{s_{u}+2, u}^{*} & =F\left(e-\gamma_{l}, k_{s_{u}+1, u}^{*}\right)+(1-\delta) k_{s_{u}+1, u}^{*}-c_{s_{u}+1, u}^{*}-\gamma_{c} \\
& =k_{s_{u}+1, u}^{*}+\left(c\left(k_{s_{u}+1}\right)\right)-c_{s_{u}+1, u}^{*} .
\end{aligned}
$$

This equation implies that $k_{s_{u}+2, u}^{*}>k_{s_{u}+1, u}^{*}$, capital increases. Since $k_{s_{u}+1}^{*}>$ $\widetilde{k}>\bar{k}$, we get from the Euler equation that $c_{s_{u}+1, u}^{*}>c_{s_{u}+2, u}^{*}$. The arrows in area $B$ of Figure 3 indicate this dynamic behavior. It follows that $\left(k_{s_{u}+2}^{*}, c_{s_{u}+2, u}^{*}\right)$ is again in area $B$ and by induction that this will be rue for every successor period. Hence the system consisting of aggregate capital and consumption moves to the south-east inside $B$, just as in the standard neoclassical growth theory. It is now straightforward that this eventually implies a contradiction to some households transversality condition, as argued in Step 2.10. 


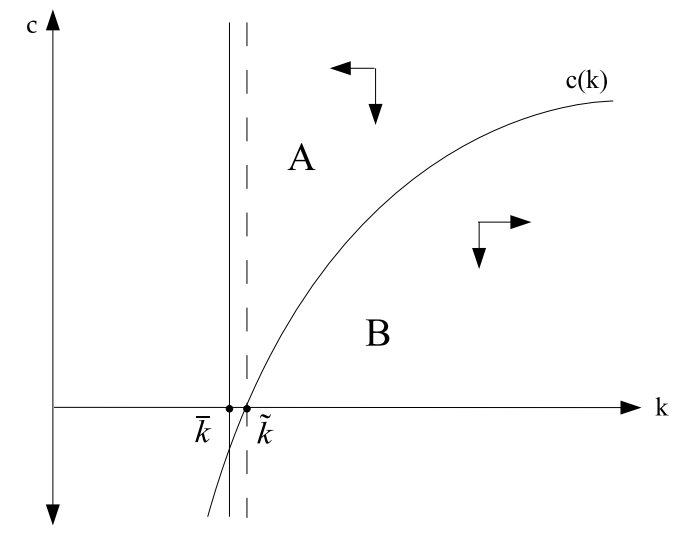

Figure 3: The Phase-Diagram in Case (ii).

Suppose now that the tuple $\left(k_{s_{u}+1}^{*}, c_{s_{u}, u}^{*}\right)$ is in area $A$. As before, we get from $c_{s_{u}, u}^{*}>c\left(k_{s_{u}+1}^{*}\right)$ that $k_{s_{u}+2, u}^{*}<k_{s_{u}+1, u}^{*}$ and from the Euler equation that $c_{s_{u}+1, u}^{*}>c_{s_{u}+2, u}^{*}$. The arrows in area $A$ of Figure 3 indicate this dynamic behavior. If $\left(k_{s_{u}+2}^{*}, c_{s_{u}+2, u}^{*}\right)$ in again in area $A$, the same result applies. If the system jumps into area $B$, we are back in the case considered in the previous paragraph. This is true for every successor period. Hence we can conclude that since the capital stock cannot diverge after entering area $B$, it must eventually converge to a value smaller $\widetilde{k}$, which leads us back to Case (i). This implies that $(*)$ cannot be true.

From $\xi_{l t, u}^{*}=\xi_{k t, u}^{*}=0$ for all $t$ and $u$ we get from Walras Law that

$$
\xi_{c t, u}^{*}+\tilde{m}_{t, u}^{*}+\tilde{n}_{t, u}^{*}=\left(\tilde{m}_{t-1, u}^{*}+\tilde{n}_{t-1, u}^{*}\right) \pi_{t-1, u}^{*}
$$

for all $t \in T$. From what we said before, we know that $\xi_{c t, u}^{*} \geq 0$ for every $u$ and $t$ which implies

$$
\tilde{m}_{t, u}^{*}+\tilde{n}_{t, u}^{*} \leq\left(\tilde{m}_{t-1, u}^{*}+\tilde{n}_{t-1, u}^{*}\right) \pi_{t-1, u}^{*}
$$

for all $t \in T$. Since this holds for all $u$, it holds in particular for all $u \geq \bar{u}$, where the existence of $\bar{u}$ comes from the previous claim. We know that there is a finite $T$ and a $0 \leq j<T$ such that real aggregate money demand in period $s_{u}+j$ is bounded away from zero by the number $c>0$. This holds for all $u \geq \bar{u}$ with the corresponding $s_{u}$. If $j \geq 1$, take a large enough $u$ and 
apply the above inequality to periods between $s_{u}$ and $s_{u}+j$ to get

$$
\begin{aligned}
\tilde{m}_{s_{u}+j, u}^{*}+\tilde{n}_{s_{u}+j, u}^{*} & \leq\left(\tilde{m}_{s_{u}-1, u}^{*}+\tilde{n}_{s_{u}-1, u}^{*}\right) \pi_{s_{u}-1, u}^{*} \cdot \ldots \cdot \pi_{s_{u}+j-1, u}^{*} \\
& =\frac{\bar{m}}{p_{s_{u}, u}^{*}} \pi_{s_{u}, u}^{*} \cdot \ldots \cdot \pi_{s_{u}+j-1, u}^{*} .
\end{aligned}
$$

The latter equality holds because the market clearing on capital, labor and consumption markets in all periods prior $s_{u}$ implies that aggregate money supply in $s_{u}$ is $\bar{m}$. If $j=0$, then it just follows that $\tilde{m}_{s_{u}+j, u}^{*}+\tilde{n}_{s_{u}+j, u}^{*} \leq$ $\frac{\bar{m}}{p_{s_{u}, u}^{*}}$. The previous claim and the definition of $s_{u}$ now imply $c<\frac{\bar{m}}{u} \pi_{s_{u}, u}^{*}$. $\ldots \cdot \pi_{s_{u}+j-1, u}^{*}$ if $j \geq 1$ and $c u<\bar{m}$ if $j=0$. The latter inequality leads immediately to a contradiction for some large enough $u$. We have shown before that the aggregate capital stock is bounded away from zero by the number $b>0$. This implies immediately that there is a finite constant $R$ which bounds the term $r_{t, u}^{*}+1-\delta$ above. Since $\pi_{t-1, u}^{*} \leq r_{t, u}^{*}+1-\delta$ it follows $c u<\bar{m} R^{j-1}$ if $j \geq 1$. For large enough $u$, this leads again to a contradiction. From this we conclude that the Step must be true.

Step $12 c_{v u}^{*} \gg 0$ for all $v$, u large enough. Hence $c_{u *}^{*} \gg 0$.

Proof. Since, for every $u$ and for every $t \in T, r_{t, u}^{*} \geq \delta$ and $w_{t, u}^{*}>0$, every single household has positive nominal wealth on the cash account in every period. Thus, the first claim is true for all finite $u$ since deflation is positive allowing for positive consumption by holding money over time between transfer dates. Since we established the existence of upper bound on monetary output prices, the second result follows.

The final Step is concerned with the convexification of households demands. The infinite dimensional character of this economy complicates the argument since we cannot simply use the theorem of Carathéodory as in finite dimensional spaces. Instead, we apply an argument given in Gale and Hellwig (1984). The interested reader may want to find more details on this problem in their paper. We will see that every $h$ indeed maximizes his utility. Combining this result with market clearing finally proves the existence of a monetary general equilibrium.

Step 13 In the limit, every agent maximizes his objective function.

Proof. In the first place, from $0 \in \overline{c o} \Xi^{h}\left(p_{u^{*}}^{*}, r_{u^{*}}^{*}, w_{u^{*}}^{*}\right)-\Xi^{J}\left(p_{u^{*}}^{*}, r_{u^{*}}^{*}, w_{u^{*}}^{*}\right)$ it follows that there is a corresponding $\xi^{h *} \in \overline{c o} \Xi^{h}\left(p_{u^{*}}^{*}, r_{u^{*}}^{*}, w_{u^{*}}^{*}\right)$. One can 
show (Rudin, 1991, Theorem 3.28) that there exists a regular Borel measure $\mu$ over the set $\Xi^{h}\left(p_{u^{*}}^{*}, r_{u^{*}}^{*}, w_{u^{*}}^{*}\right)$ such that

$$
\xi^{h *}=\int_{\Xi^{h}\left(p_{u^{*}}^{*}, r_{u^{*}}^{*}, w_{u^{*}}^{*}\right)} \xi \mu(d \xi) .
$$

Recall that $H=[0,1]$ and let $\lambda$ be the Lebesgue measure defined on the Borel sets of $H$. The theorem of Skorokhod implies that, for the space $\Xi^{h}\left(p_{u^{*}}^{*}, r_{u^{*}}^{*}, w_{u^{*}}^{*}\right)$ and the Borel measure $\mu$, there exists a measurable mapping $g:[0,1] \rightarrow \Xi^{h}\left(p_{u^{*}}^{*}, r_{u^{*}}^{*}, w_{u^{*}}^{*}\right)$ such that $\mu=\lambda \circ g^{-1}$. Using this and a change of variable, we get

$$
\int_{\Xi^{h}\left(p_{u^{*}}^{*}, r_{u^{*}}^{*}, w_{u^{*}}^{*}\right)} \xi \mu(d \xi)=\int_{\Xi^{h}\left(p_{u^{*}}^{*}, r_{u^{*}}^{*}, w_{u^{*}}^{*}\right)} \xi\left(\lambda \circ g^{-1}\right)(d \xi)=\int_{0}^{1} g(h) d h .
$$

By combining (24) and (25),

$$
\xi^{h *}=\int_{0}^{1} g(h) d h
$$

with $g(h) \in \Xi^{h}\left(p_{u^{*}}^{*}, r_{u^{*}}^{*}, w_{u^{*}}^{*}\right)$ for all $h \in H$. By definition of $\Xi^{h}$, there is a vector $\sigma_{u^{*}}^{h *} \in \bar{\phi}^{h *}\left(p_{u^{*}}^{*}, r_{u^{*}}^{*}, w_{u^{*}}^{*}\right)$ which corresponds to $g(h) \in \Xi^{h}\left(p_{u^{*}}^{*}, r_{u^{*}}^{*}, w_{u^{*}}^{*}\right)$ for almost every $h \in H$. Hence (almost) every household maximizes his utility.

The interpretation of the argument given is straightforward: the measure $\mu$ is the distribution of individual demands over the set $\Xi^{h}\left(p_{u^{*}}^{*}, r_{u^{*}}^{*}, w_{u^{*}}^{*}\right)$ and the function $g$ assigns the demand $g(h) \in \Xi^{h}\left(p_{u^{*}}^{*}, r_{u^{*}}^{*}, w_{u^{*}}^{*}\right)$ to agent $h \in H=[0,1]$.

\section{References}

[1] Aliprantis, C. and Border, K., 1999, Infinite Dimensional Analyis - A Hitchhiker's Guide, Second Edition, Springer Verlag, Heidelberg.

[2] Alvarez, F., Atkeson, A. and Edmond, C., 2003, On the sluggish Response of Prices to Money in an inventory-theoretic Model of Money Demand, NBER Working Paper 10016.

[3] Baumol, W.J., 1952, The Transactions Demand for Cash: An inventorytheoretic Approach, Quarterly Journal of Economics, 66, 545-556. 
[4] Chatterjee, S., Corbae, D., 1992, Endogenous Market Participation and the General Equilibrium Value of Money, Journal of Political Economy, $100,615-646$.

[5] Dréze, J. and Polemarchakis, H., 2000, Monetary Equilibria, in: Debreu, G., Neuefeind, W., Trockel, W. (eds.), Economic Essays - A Festschrift for Werner Hildenbrand, Springer, Berlin.

[6] Folland, G.B. (1999), Real Analysis: Modern Tequniques and their Applications, second edition, New York: John Wiley.

[7] Gale, D. and Hellwig, M.F., 1984, A General Equilibrium Model of the Transactions Demand for Money, CARESS Working Paper 85/07.

[8] Gale, D. and Hellwig, M.F., 1988, A General Equilibrium Model of the Transactions Demand for Money, mimeo.

[9] Ghiglino, C., 2004, Wealth Inequality and Dynamic Stability, forthcomingin the Journal of Economic Theory.

[10] Grossman, S., 1987, Monetary Dynamics with Proportional Transactions Costs and fixed Payment Periods, in: Barnett, W., Singleton, K.J. (eds.), New Approaches to Monetary Dynamics, Canbridge, University Press.

[11] Grossman, S., Weiss, L., 1983, A Transaction Based Model of the Monetary Transmission Mechanism, American Economic Review, 88, 1-32.

[12] Hellwig, M.F., 1993, The Challenge of Monetary Theory, European Economic Review 37, 215-242.

[13] Jovanovic, B., 1982, Inflation and Welfare in the Steady State, Journal of Political Economy 90, 561-577.

[14] Romer, D. , 1986, A simple General Equilibrium Version of the BaumolTobin model, Quaterly Journal of Economics 101, 663-686.

[15] Rotemberg, J. , 1984, A Monetaqry Equilibrium Model with Transactions Costs, Journal of Political Economy 92, 40-58.

[16] Rudin, W. , Functional Analysis, Second Edition, McGraw Hill, New York, 1991.

[17] Tobin, J., 1956, The Interest Elasticity of the Transactions Demand for Cash, Review of Economics and Statistics 88, 241-247. 
[18] Zeira, J., 2005, Money and the Size of Transactions, CEPR-Discussion Paper No. 5010. 